\title{
Fixed-point elimination in the Intuitionistic Propositional Calculus
}

\author{
SILVIO GHILARDI, Dipartimento di Matematica, Università degli Studi di Milano, Italy \\ MARIA JOÃO GOUVEIA, Faculdade de Ciências da Universidade de Lisboa, Portugal \\ LUIGI SANTOCANALE, LIS, CNRS UMR 7020, Aix-Marseille Université, France
}

It follows from known results in the literature that least and greatest fixed-points of monotone polynomials on Heyting algebras-that is, the algebraic models of the Intuitionistic Propositional Calculus-always exist, even when these algebras are not complete as lattices. The reason is that these extremal fixed-points are definable by formulas of the IPC. Consequently, the $\mu$-calculus based on intuitionistic logic is trivial, every $\mu$-formula being equivalent to a fixed-point free formula. In the first part of this paper we give an axiomatization of least and greatest fixed-points of formulas, and an algorithm to compute a fixed-point free formula equivalent to a given $\mu$-formula. The axiomatization of the greatest fixed-point is simple. The axiomatization of the least fixed-point is more complex, in particular every monotone formula converges to its least fixed-point by Kleene's iteration in a finite number of steps, but there is no uniform upper bound on the number of iterations. The axiomatization yields a decision procedure for the $\mu$-calculus based on propositional intuitionistic logic. The second part of the paper deals with closure ordinals of monotone polynomials on Heyting algebras and of intuitionistic monotone formulas; these are the least numbers of iterations needed for a polynomial/formula to converge to its least fixed-point. Mirroring the elimination procedure, we show how to compute upper bounds for closure ordinals of arbitrary intuitionistic formulas. For some classes of formulas we provide tighter upper bounds that, in some cases, we prove exact.

\section{ACM Reference Format:}

Silvio Ghilardi, Maria João Gouveia, and Luigi Santocanale. 2019. Fixed-point elimination in the Intuitionistic Propositional Calculus. ACM Trans. Comput. Logic 1, 1 (April 2019), 38 pages. https://doi.org/10.1145/nnnnnnn.nnnnnnn

\section{INTRODUCTION}

The original motivation for developing the research that we present in this paper was the investigation of $\mu$-calculi based on Intuitionistic Logic. A $\mu$-calculus [Arnold and Niwiński 2001] is a prototypical kind of computational logic, obtained from a base logic or a base algebraic system, by adding distinct forms of iteration, least fixed-points and greatest fixed-points, so to increase expressivity. We ended up studying fixed-points within Intuitionistic Logic mostly by observing structural similarities between the propositional modal $\mu$-calculus and the Intuitionistic Propositional Calculus (IPC). Bisimulation quantifiers (also known as uniform interpolants) within the propositional modal $\mu$-calculus were studied in [D'Agostino and Hollenberg 2000]; in this work a formula built by using these kind of quantifiers was employed to prove that PDL (Propositional Dynamic Logic, see [Harel et al. 2000]) lacks the uniform interpolation property. In [Pitts 1992] the author discovered that IPC also has bisimulation quantifiers; together with the deduction

This is an extended version of the conference paper [Ghilardi et al. 2016]. Partially supported by the "LIA LYSM AMU CNRS ECM INdAM".

Authors' addresses: Silvio Ghilardi, Dipartimento di Matematica, Università degli Studi di Milano, Italy, silvio.ghilardi@unimi.it; Maria João Gouveia, Faculdade de Ciências da Universidade de Lisboa, Portugal, mjgouveia@fc.ul.pt; Luigi Santocanale, LIS, CNRS UMR 7020, Aix-Marseille Université, France, luigi.santocanale@lif.univ-mrs.fr.

Permission to make digital or hard copies of all or part of this work for personal or classroom use is granted without fee provided that copies are not made or distributed for profit or commercial advantage and that copies bear this notice and the full citation on the first page. Copyrights for components of this work owned by others than ACM must be honored. Abstracting with credit is permitted. To copy otherwise, or republish, to post on servers or to redistribute to lists, requires prior specific permission and/or a fee. Request permissions from permissions@acm.org.

(c) 2019 Association for Computing Machinery.

Manuscript submitted to ACM 
property, uniform interpolants give a rather strong structure to the category of (finitely presented) Heyting algebras (the algebraic models of the IPC); this structure was axiomatized and studied in [Ghilardi and Zawadowski 1997, 2011]. Quantified formulas analogous to the one of [D’Agostino and Hollenberg 2000] make sense in every category with this structure and they indeed define the extremal fixed-points of monotone formulas. This made us conjecture that a $\mu$-calculus based on Intuitionistic Logic is trivial, meaning that every $\mu$-formula is equivalent to a fixed-point free formula. The conjecture actually holds because of a deep result in Intuitionistic Logic. It was proved in [Ruitenburg 1984] that, for each formula $\phi(x)$ of the IPC, there exists a number $n \geq 0$ such that $\phi^{n}(x)$-the formula obtained from $\phi$ by iterating $n$ times substitution of $\phi$ for the variable $x$-and $\phi^{n+2}(x)$ are provably equivalent in Intuitionistic Logic. An immediate corollary of this result is that a syntactically monotone formula $\phi(x)$ converges both to its least fixed-point and to its greatest fixed-point in at most $n$ steps. We write $\mu_{x} \cdot \phi(x)=\phi^{n}(\perp)$ and $v_{x} . \phi(x)=\phi^{n}(\top)$ to express this fact, using a modern notation based on $\mu$-calculi. These two identities can be used to argue that every formula of a $\mu$-calculus based on Intuitionistic Logic is equivalent to a fixed-point free formula.

Ruitenburg's work leaves open how to compute or estimate the least number $n$ such that $\phi^{n}(x)=\phi^{n+2}(x)$-we shall call such a number the Ruitenburg's number of $\phi$ and denote it by $\rho(\phi)$. As our motivations stem from fixed-point theory and $\mu$-calculi, we remark that being able to compute or bound Ruitenburg's number $\rho(\phi)$ might yield an overapproximation of the least integer $k$ such that $\mu_{x} . \phi(x)=\phi^{k}(\perp)$-we call such a number $k$ closure ordinal of $\phi$. For example, when considering the dual analogous problem, and so the greatest fixed-point of $\phi$, we shall see that the least number $k$ such that of $\phi$ is $v_{x} . \phi(x)=\phi^{k}(T)$ is 1 at most, while $\rho(\phi)$ can be arbitrarily large. Least fixed-points over Intuitionistic Logic have also been considered in [Mardaev 1993]. In op. cit. the author gave an independent proof that least fixed-points of monotone intuitionistic formulas are definable. His proof relies on semantics methods and on the coding of Intuitionistic Logic into Grzegorczyk's Logic; the proof was further refined in [Mardaev 1994] to encompass the standard coding of Intuitionistic Logic into its modal companion, the logic S4.

The results presented in this paper are also part of a line of research that we are currently exploring, and that lead us to studying fixed-points within Intuitionistic Logic. We aim at identifying, under a unified perspective, reasons that make alternation-depth hierarchies of $\mu$-calculi degenerate or trivial. A $\mu$-calculus is obtained by adding formal least and greatest fixed-points to an underlying logical-algebraic system, so it generates formula-terms with nested extremal fixed-points. The alternation-depth hierarchy [Arnold and Niwiński 2001, §2.6] of a $\mu$-calculus measures the complexity of a formula-term, as a function of the nesting of the different types of fixed-points and with respect to a fixed class of models. It is well known that fixed-points that are unguarded can be eliminated in the propositional modal $\mu$-calculus [Kozen 1983]. This fact can be rephrased by saying that the alternation-depth hierarchy of the $\mu$-calculus over distributive lattices is trivial (every $\mu$-term is equivalent to a fixed-point free term). To closely understand and to refine this result was one of the goals of [Frittella and Santocanale 2014]. In that paper the authors were able to exhibit equational classes of lattices $\mathcal{D}_{n}$-with $\mathcal{D}_{0}$ the class of distributive lattices-where the extremal fixed-points can be uniformly computed by iterating a formula-term $n+1$ times from the bottom/top of the lattice; moreover, they showed that these uniform upper bounds are optimal. For those classes of lattices, the degeneracy of the alternation-depth hierarchy originates in the structure of the lattices in the class. The next and most natural algebraic setting extending distributive lattices and where to study fixed-points, was given by Heyting algebras and Intuitionistic Logic.

This paper is divided in two parts. In the first part, we firstly show how to eliminate greatest fixed-points. Namely we argue that, for every intuitionistic formula $\phi(x)$ with the specified variable $x$ positive in $\phi(x), v_{x} \cdot \phi(x)=\phi(T)$. Greatest fixed-points of intuitionistic formulas are reached from the top of the lattice after one iteration, exactly as 
in the case of distributive lattices. At a second stage we present the elimination procedure of least fixed-points; the procedure yields, for every formula $\phi(x)$ as above, a (fixed-point free) intuitionistic formula $\psi$ such that $\mu_{x} . \phi(x)=\psi$. The two elimination procedures can be cast into a procedure that yields a fixed-point free formula equivalent to an arbitrary formula of the $\mathbf{I P C}_{\mu}$, the $\mu$-calculus based on Intuitionistic Logic. Since IPC is decidable, the procedure also provides a decision procedure for the IPC ${ }_{\mu}$. Even if elimination of greatest fixed-points turns out to be somewhat trivial, it plays an important role for eliminating least fixed-points. Natural properties of fixed-points lead to identify two orthogonal syntactic fragments of the IPC: we call the formulas belonging to these fragments weakly negative and strongly positive, respectively. Least fixed-point elimination is split between two kind of eliminations, one for each fragment. For weakly negative formulas, elimination of least fixed-points is a consequence of greatest fixed-point elimination. Least fixed-point elimination for strongly positive formulas relies on these formulas being inflating (i.e., semantically they give rise to inflating monotone functions) and other ingredients.

The second part of the paper studies closure ordinals of intuitionistic positive formulas. The closure ordinal of $\phi(x)$-which, we recall, is the least integers $n$ for which we can write $\mu_{x} . \phi(x)=\phi^{n}(\perp)$-yields a representation of the least fixed-point $\mu_{x} . \phi(x)$ alternative to the one presented in the first part. Such representation can be exploited notationally, as in $\mu$-calculi with explicit approximations [Dam and Gurov 2002], computationally, because of its reduced space requirements, at least if variable sharing is used, and also axiomatically. We firstly present general results for producing upper bounds of closure ordinals of monotone functions and then we add results that are specific for Heyting algebras and Intuitionistic Logic. Whenever it is possible, we also argue that those bounds are tight. By combining these results and, at the same time, by paralleling the least fixed-point elimination procedure, upper bounds of closure ordinals of formula-terms $\phi(x)$ can be computed. It turns out that these bounds are not tight. We focus therefore on closure ordinals of strongly positive formula-terms that, in view of tightness of bounds, are the most problematic. We produce specific (and better) bounds for these formulas; in this case our proof yields bounds on Ruitenburg's numbers and so also new insights on his theorem. We finish the second part of the paper by presenting a syntactic fragment (formulas in the fragment are disjunctions of what we call almost-topologies) and prove a surprising fact: closure ordinals of formulas in this fragment have 3 as a uniform upper bound.

Comparing the present work to our previous results on degeneracies of alternation-depth hierarchies, reasons for degeneracies appear now to have a very different nature. Several are the ingredients contributing to the existence of a finite closure ordinal of every intuitionistic formula, thus to the degeneracy of the alternation-depth hierarchy of the $\mu$-calculus based on Intuitionistic Logic. Probably the most important among them is strongness of monotone polynomials on Heyting algebras. The naming comes from category theory: a monotone polynomial $f: H \longrightarrow H$ (with $H$ a Heyting algebra) is strong if it has a strength; in turn, this is equivalent to say that, as a functor, it is enriched over the closed category $H$ [Kelly 1982; Kock 1972]. Yet strongness is just a possible naming for a general logical phenomenon, the capability of an equational theory to partly encode quasi-equations. On the proof-theoretic side, this phenomenon is known as the deduction theorem; on the algebraic side it translates to equationally definable principal congruences [Blok et al. 1984]. In modal logic the deduction theorem is equivalent to having a master modality [Kracht 2006, Theorem 64]; as a matter of fact, having a master modality appears to be a common pattern in several works on alternation-depth hierarchies modal $\mu$-calculi [Alberucci and Facchini 2009a; Bertrand and Schnoebelen 2013; D’Agostino and Lenzi 2010; Mardaev 1994, 2007]. Other ingredients are the following. For some polynomials, existence and finiteness of the closure ordinal is a consequence of being inflating (or expanding) and, on the syntactic level, to a restriction to the use of conjunction that determines a notion of disjunctive formula. A key ingredient of the algorithm 
we present is creation of least fixed-points via the Rolling equation (cf. Lemma 1), a fact already used in [D'Agostino and Lenzi 2010]. For Intuitionistic Logic and Heyting algebras, where formula-terms can be semantically antitone (i.e. contravariant), existing greatest fixed-points create least fixed-points. Overall the most striking difference with the case of distributive lattices and generalizations of distributive lattices [Frittella and Santocanale 2014] is the absence of a finite uniform upper bound on the closure ordinals, the rate of convergence to the least fixed-point crucially depends on the size and shape of the formula.

The considerations that we shall develop rely on well-known equivalences of fixed-point expressions [Arnold and Niwiński 2001; Bloom and Ésik 1993]. This distinguishes our approach from previous works [Mardaev 1993; Ruitenburg 1984]. Using these equivalences we can move the focus from existence and definability of fixed-points in Intuitionistic Logic towards the explicit construction of them. On the way, let us remark that the simple characterization of greatest fixed-points in Intuitionistic Logic $v_{x} . \phi(x)=\phi(T)$, that yet plays an important role in the elimination procedure of least fixed-points, appears to be orthogonal to Ruitenburg's work, while greatest fixed-points are not considered in Mardaev's work. For the modal $\mu$-calculus, a similar algorithmic approach to fixed-point elimination appears in [Lehtinen and Quickert 2015].

The paper is organized as follows. The goal of the first part, Sections 2 to 6 , is to present the fixed-point elimination procedure for the Intuitionistic Propositional Calculus. We recall in Section 2 some elementary facts from fixed-point theory. In Section 3 we present the Intuitionistic Propositional Calculus and introduce its fixed-point extension, the Intuitionistic Propositional $\mu$-Calculus. In Section 4 we pinpoint strongness, a property of monotone functions on Heyting algebras that will be pervasive in all the paper. We prove some elementary facts about strong functions and their least fixed-points and give a simple axiomatization of their greatest fixed-points. In Section 5 we digress on bisimulation quantifiers and argue that the existence of extremal fixed-points can be inferred from these quantifiers. Section 6 presents the elimination procedure.

The second and last part of the paper starts with Section 7 and deals with estimating closure ordinals of formula-terms of the IPC. We begin by presenting some general results, that apply to arbitrary monotone functions on posets with a least element. In the second half of Section 7 we present some results specific to Heyting algebras; the results from this Section are sufficient to estimate an upper bound of the closure ordinal of any formula-term, yet these upper bounds are not tight. Therefore we estimate in Section 8 closure ordinals of conjunctions of disjunctive formulas (defined in Section 6) which, in view of tightness of upper bounds, appear to be the most difficult. Our work actually yields upper bounds of Ruitenburg's numbers of these formulas and a closed expression for the formula $\phi^{\rho(\phi)}$ (when $\phi$ is such a disjunction and where $\rho(\phi)$ is the Ruitenburg's number of $\phi$ ). In Section 9 we exemplify how the search for bounds of closure ordinals leads to some non-trivial discovery: we present an infinite family of formula-terms that-while being more and more complex-uniformly converge to their least fixed-point in 3 steps. We add concluding remarks in Section 10 .

Manuscript submitted to ACM 
Contents

\begin{tabular}{|c|c|c|}
\hline \multicolumn{2}{|c|}{ Abstract } & 1 \\
\hline 1 & Introduction & 1 \\
\hline \multicolumn{2}{|c|}{ Contents } & 5 \\
\hline 2 & Elementary fixed-point theory & 5 \\
\hline 3 & The Intuitionistic Propositional $\mu$-Calculus & 6 \\
\hline 4 & Strong monotone functions and fixed-points & 8 \\
\hline 5 & Bisimulation quantifiers and fixed-points & 10 \\
\hline 6 & The elimination procedure & 12 \\
\hline 6.1 & Summary of the procedure & 13 \\
\hline 6.2 & Computation of a normal form & 13 \\
\hline 6.3 & Strongly positive elimination & 14 \\
\hline 6.4 & Weakly negative elimination & 16 \\
\hline 7 & Upper bounds for closure ordinals & 17 \\
\hline 7.1 & General results & 17 \\
\hline 7.2 & Results for Heyting algebras & 20 \\
\hline 8 & Ruitenburg's numbers for strongly positive formulas & 23 \\
\hline 8.1 & The support of a formula & 25 \\
\hline 8.2 & Word formulas & 25 \\
\hline 8.3 & Conjunctions of star formulas & 27 \\
\hline 8.4 & A game for iterated conjunctions of star formulas & 30 \\
\hline 9 & A constant upper bound for disjunctions of almost-topologies & 32 \\
\hline 9.1 & Elementary properties of atops & 33 \\
\hline 9.2 & Closure of prefixed-points of strong monotone mappings under exponentiation & 34 \\
\hline 9.3 & Convergence in 3 steps for disjunctions of atops & 34 \\
\hline 10 & Conclusions & 36 \\
\hline
\end{tabular}

\section{ELEMENTARY FIXED-POINT THEORY}

Let $P$ and $Q$ be posets. A function $f: P \longrightarrow Q$ is monotone if $x \leq y$ implies $f(x) \leq f(y)$, for each $x, y \in P$. If $f: P \longrightarrow P$ is a monotone endofunction, then $x \in P$ is a prefixed-point of $f$ if $f(x) \leq x$; we denote by Pre $_{f}$ the set of prefixed points of $f$. Whenever Pre $_{f}$ has a least element, we denote it by $\mu$. $f$. Therefore, $\mu$.f denotes the least prefixed-point of $f$, whenever it exists. If $\mu$. $f$ exists, then it is a fixed-point of $f$, necessarily the least one. The notions of least prefixed-point and of least fixed-point coincide on complete lattices or when the least fixed-point is computed by iterating from the bottom of a lattice; for our purposes they are interchangeable, so we shall abuse of language and refer to $\mu . f$ as the least fixed-point of $f$. Dually (and abusing again of language), the greatest fixed-point of $f$ shall be denoted by $v$. $f$.

Let us mention some well known identities from fixed-point theory, see for example [Bloom and Ésik 1993] or [Arnold and Niwiński 2001]. Notice however that the statements that we present below also assert and emphasize the 
existence of some least fixed-point-we do not assume completeness of the posets. Full proofs of these statements can be found in [Santocanale 2002].

Lemma 1. Let $P, Q$ be posets, $f: P \longrightarrow Q$ and $g: Q \longrightarrow P$ be monotone functions. If $\mu .(g \circ f)$ exists, then $\mu .(f \circ g)$ exists as well and is equal to $f(\mu .(g \circ f))$.

As we do not work in complete lattices (so we are not ensured that least fixed-points exist) we express the above statement via the equality

$$
\mu .(f \circ g):=f(\mu .(g \circ f))
$$

where the colon emphasizes existence: if the least fixed-point in the expression on the right exists, then this expression is the least fixed-point of $f \circ g$. Analogous notations will be used later. We endow the product of two posets $P$ and $Q$ with the coordinatewise ordering. Therefore a function $f: P \times Q \longrightarrow R$ is monotone if, as a function of two variables, it is monotone in each variable. To deal with least fixed-points of functions of many variables, we use the standard notation: for example, if $f: P \times P \longrightarrow P$ is the monotone function $f(x, y)$, then, for a fixed $p \in P, \mu_{x} . f(x, p)$ denotes the least fixed-point of $f(x, p)$. Let us recall that the correspondence $p \mapsto \mu_{x} . f(x, p)$-noted $\mu_{x} . f(x, y)$-is again monotone.

Lemma 2. If $P$ is a poset and $f: P \times P \longrightarrow P$ is a monotone mapping, then

$$
\mu_{x} \cdot f(x, x):=\mu_{x} \cdot \mu_{y} \cdot f(x, y) .
$$

Again, the expression above shall be read by saying that if $\mu_{y} \cdot f(x, y)$ exists, for each $x \in P$, and if $\mu_{x} \cdot \mu_{y} \cdot f(x, y)$ exists, then $\mu_{x} . f(x, x)$ exists as well and is given by the expression on the right.

Recall that a function $f$ from $A$ to a product $B \times C$ is uniquely determined by two functions $g: A \longrightarrow B$ and $h: A \longrightarrow C$ via composing with projections; we therefore write $f=\langle g, h\rangle$ and use a similar notation for products with more factors.

Lemma 3. If $P$ and $Q$ are posets and $\langle f, g\rangle: P \times Q \longrightarrow P \times Q$ is a monotone function (so $f: P \times Q \longrightarrow P$ and $g: P \times Q \longrightarrow Q)$, then $\mu .\langle f, g\rangle:=\left\langle\mu_{1}, \mu_{2}\right\rangle$, where

$$
\mu_{1}=\mu_{x} \cdot f\left(x, \mu_{y} \cdot g(x, y)\right) \quad \text { and } \quad \mu_{2}=\mu_{y} \cdot g\left(\mu_{1}, y\right) .
$$

\section{THE INTUITIONISTIC PROPOSITIONAL $\mu$-CALCULUS}

Formulas of the Intuitionistic Propositional Calculus (IPC) are generated according to the following grammar:

$$
\phi \Rightarrow x|\top| \phi \wedge \phi|\perp| \phi \vee \phi \mid \phi \rightarrow \phi,
$$

where $x$ ranges over a countable set $\mathbb{X}$ of propositional variables. The set of these formulas shall be denoted $\mathcal{F}_{\text {IPC. }}$ The consequence relation of the IPC, relating a set of formulas to a formula, is described by means of the intuitionistic sequent calculus, Gentzen's system LJ [Gentzen 1935], see also [Negri and von Plato 2001]. Therefore we shall write $\left.\Gamma\right|_{\mathbf{L J}} \delta$ if the sequent $\Gamma \vdash \delta$ is derivable in the system $\mathbf{L J}$.

It is well known that the IPC is sound and complete w.r.t. the class of its algebraic models, the Heyting algebras that we introduce next.

Manuscript submitted to ACM 
Definition 1. A Heyting algebra $H$ is a bounded lattice (so $H$ has a least element $\perp$ and a greatest element $T$ ) equipped with a binary operation $\rightarrow$ such that the following equations hold in $H$ :

$$
\begin{aligned}
x \wedge(x \rightarrow y) & =x \wedge y, & & x \wedge(y \rightarrow x)=x, \\
x \rightarrow x & =\top, & x & \rightarrow(y \wedge z)=(x \rightarrow y) \wedge(x \rightarrow z) .
\end{aligned}
$$

We can define on any Heyting algebra a partial order by saying that $x \leq y$ holds when $x \vee y=y$. We identify formulas of the IPC with terms of the theory of Heyting algebras, constructed therefore from variables and using the signature $\langle T, \wedge, \perp, \vee, \rightarrow\rangle$; we shall therefore refer to objects generate by the grammar (1) as formula-terms. ${ }^{1}$ Let $\phi$ be such a formula-term, let $H$ be a Heyting algebra and let $v: \mathbb{X} \longrightarrow H$ be a valuation of the propositional variables in $H$; we write $\llbracket \phi \rrbracket_{v}$ for the result of evaluating the formula in $H$, starting from the variables (the definition of $\llbracket \cdot \rrbracket_{v}$ is given as usual by induction). The soundness and completeness theorem of the IPC over Heyting algebras, see e.g. [Bezhanishvili and de Jongh 2006], can then be stated as follows:

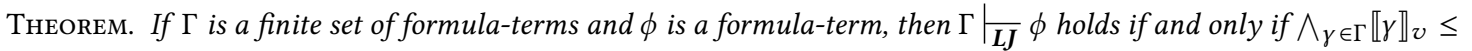
$\llbracket \phi \rrbracket_{v}$ holds, in every Heyting algebra $H$ and for every valuation of the propositional variables $v: \mathbb{X} \longrightarrow H$.

Given this theorem, we shall often abuse of notation and write $\leq$ in place of $\left.\right|_{\mathbf{L J}}$, and the symbol $\overline{\mathrm{IPC}}$ (or even the equality symbol =) to denote provable equivalence of formulas. That is to say, we identify formula-terms with elements of the Lindenbaum-Tarski algebra of the IPC. Recall that this algebra is also the free Heyting algebra over the set $\mathbb{X}$ of propositional variables; therefore we shall denote it by $\mathrm{F}_{\mathrm{H}}[\mathbb{X}]$. More generally, for a set of generators $Y$, the free Heyting algebra on this set shall be denoted by $\mathrm{F}_{\mathrm{H}}[Y]$.

We aim at studying extremal fixed-points on Heyting algebras and so we formalize next the Intuitionistic Propositional $\mu$-Calculus $\left(\mathrm{IPC}_{\mu}\right)$.

An occurrence of a variable $x$ is positive in a formula-term $\phi$ if, in the syntax tree of $\phi$, the path from the root to the leaf labeled by this variable occurrence contains an even number of nodes such that: (i) they are labeled by a subformula $\psi_{1} \rightarrow \psi_{2}$ and (ii) their immediate successor on the path is the left son labeled by the subformula $\psi_{1}$. If on this path the number of those nodes is odd, then we say that this occurrence of $x$ is negative in $\phi$. For example, in the formula-term $((x \rightarrow y) \rightarrow(x \vee z)) \rightarrow w$ the first occurrence of $x$ is positive but the second occurrence is negative. A variable $x$ is positive in a formula $\phi$ if each occurrence of $x$ is positive in $\phi$. A variable $x$ is negative in a formula $\phi$ if each occurrence of $x$ is negative in $\phi$. We enrich the grammar (1) with the following two productions:

$$
\phi \Rightarrow \mu_{x} \cdot \phi, \quad \phi \Rightarrow v_{x} \cdot \phi
$$

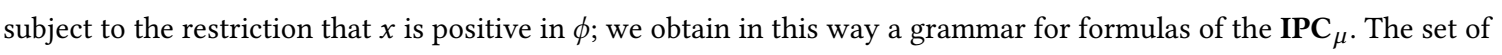
formulas generated by this grammar shall be denoted by $\mathcal{F}_{\mathrm{IPC}_{\mu}}$. Notice that the symbols $\mu$ and $v$ syntactically behaves as binders (similar to quantifiers), so the notions of free and bound variable in a formula-term is defined as usual.

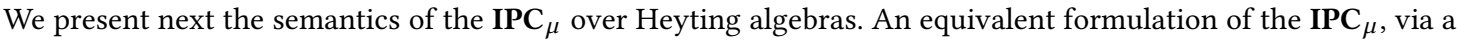
sequent calculus, appears in [Clairambault 2013, §2].

For a formula $\phi$ of the $\mathbf{I P C}_{\mu}$, let $\mathbb{X}_{\phi}$ denote the set of variables having a free occurrence in $\phi$. Let $H$ be a Heyting algebra (that we do not suppose complete); we define next a partial evaluation function sending $\phi \in$ IPC $_{\mu}$ and $v: \mathbb{X}_{\phi} \longrightarrow H$ to $\llbracket \phi \rrbracket_{v}$. We only cover the cases of formulas $\mu_{x} . \phi$ and $v_{x} . \phi$, since the other cases are the usual ones.

${ }^{1}$ In view of the verbosity of the naming formula-terms we shall often use formula or term as a synonym of formula-term.

Manuscript submitted to ACM 
Thus let $\phi$ be a formula of the $\mathbf{I P C}_{\mu}$, let $x$ be positive in $\phi$, and suppose that $\llbracket \phi \rrbracket_{u}$ is defined, for each $u: \mathbb{X}_{\phi} \longrightarrow H .^{2}$ If $v: \mathbb{X}_{\phi} \backslash\{x\} \longrightarrow H$ is a valuation of all the free variables of $\phi$ but $x$, then $(v, h / x): \mathbb{X}_{\phi} \longrightarrow H$ is the valuation such that $(v, h / x)(x)=h$ and $(v, h / x)(y)=v(y)$ for $y \neq x$. Since $x$ is positive in $\phi$, then the function

$$
\llbracket \phi \rrbracket_{v}: H \longrightarrow H, \quad h \mapsto \llbracket \phi \rrbracket_{(v, h / x)}
$$

is monotone; therefore, if the extremal fixed-points of this function exist, ${ }^{3}$ then we define

$$
\llbracket \mu_{x} \cdot \phi \rrbracket_{v}=_{\text {def }} \mu \cdot \llbracket \phi \rrbracket_{v}, \quad \llbracket v_{x} \cdot \phi \rrbracket_{v}=_{\text {def }} v \cdot \llbracket \phi \rrbracket_{v} .
$$

Clearly, when $H$ is a complete Heyting algebra, then extremal fixed-points of monotone functions exists, so the correspondence $(\phi, v) \mapsto \llbracket \phi \rrbracket_{v}$ is total. We argue next that this correspondence is always total.

We say that two formulas $\phi$ and $\psi$ of the $\mathbf{I P C}_{\mu}$ are equivalent over Heyting algebras if, for each Heyting algebra $H$ and each $v: \mathbb{X}_{\phi} \cup \mathbb{X}_{\psi} \longrightarrow H, \llbracket \phi \rrbracket_{v}$ is defined if and only if $\llbracket \psi \rrbracket_{v}$ is defined, and $\llbracket \phi \rrbracket_{v}=\llbracket \psi \rrbracket_{v}$ whenever they are both defined. We write $\phi=\overline{\mathbf{I P C}}_{\mu} \psi$ when two formulas $\phi$ and $\psi$ of the $\mathbf{I P C}_{\mu}$ are equivalent over Heyting algebras.

Let us say that a formula $\phi$ of $\mathbf{I P C}_{\mu}$ is fixed-point free if it does not contain either of the symbols $\mu, v$ (that is, if it is a formula of the IPC).

PROPOSITION 4. Every formula $\phi$ of the IPC $\boldsymbol{I P}_{\mu}$ is equivalent over Heyting algebras to a fixed-point free formula $\chi$. In particular $\llbracket \phi \rrbracket_{v}$ is defined, for each formula-term $\phi$ of the $\mathbf{I P C}_{\mu}$, each Heyting algebra $H$, and each $v: \mathbb{X}_{\phi} \longrightarrow H$,

Proof. Clearly, the first statement of the Proposition holds if we can show that it holds whenever $\phi=\mu_{x} \cdot \psi$ or $\phi=v_{x} . \psi$, where $\psi$ is a fixed-point free formula. For a natural number $n \geq 0$, let $\psi^{n}(x)$ denote the formula obtained by substituting $x$ for $\psi n$ times. Ruitenburg [Ruitenburg 1984] proves that, for each intuitionistic propositional formula $\psi$, there exists a number $n \geq 0$ such that the formulas $\psi^{n}(x)={ }_{\text {IPC }} \psi^{n+2}(x)$. If $x$ is positive in $\psi$, then instantiating $x$ with $\perp$, leads to the equivalence $\psi^{n+1}(\perp)={ }_{\text {IPC }} \psi^{n}(\perp)$. Yet this relation enforces $\psi^{n}(\perp)$ to be the least fixed-point of $\psi$, namely $\llbracket \mu_{x} \cdot \psi \rrbracket_{v}=\llbracket \psi^{n}(\perp) \rrbracket_{v}$ for each $H$ and $v: \mathbb{X}_{\psi} \longrightarrow H$. That is, we have $\mu_{x} \cdot \psi={ }_{\mathbf{I P C}_{\mu}} \psi^{n}(\perp)$; similarly, we get $v_{x} \cdot \psi \underset{{ }_{\mathrm{IPC}}}{ } \psi^{n}(\mathrm{~T})$.

According to the Proposition (and to Ruitenburg's result [Ruitenburg 1984]) the expansion of the IPC with extremal fixed-points does not increase its expressive power. This does not exclude the use of $\mathbf{I P C}_{\mu}$ as a convenient formalism, but raises the problem of (efficiently) computing, for each $\phi \in \mathcal{F}_{\mathbf{I P C}_{\mu}}$, a formula $\psi \in \mathcal{F}_{\text {IPC }}$ such that $\phi={ }_{\mathbf{I P C}_{\mu}} \psi$.

For a formula $\mu_{x}$. $\phi$ with $\phi$ fixed-point free, this can be achieved by computing the Ruitenburg's numbers $\rho(\phi)$. An attentive reading of Ruitenburg's paper shows that $\rho(\phi) \leq 2 n+2$ where $n$ is the size of the formula. Yet, $\rho(\phi)$ might not

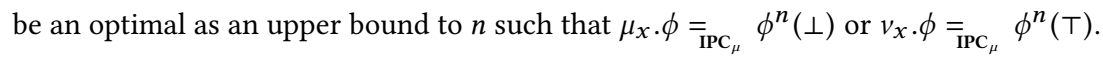

\section{STRONG MONOTONE FUNCTIONS AND FIXED-POINTS}

If $H$ is a Heyting algebra and $f: H \longrightarrow H$ is any function, then $f$ is said to be compatible if

$$
x \wedge f(y)=x \wedge f(x \wedge y), \quad \text { for any } x, y \in H .
$$

\footnotetext{
${ }^{2}$ If, for some $u: \mathbb{X}_{\phi} \longrightarrow H, \llbracket \phi \rrbracket_{u}$ is not defined, then $\llbracket \mu_{x} . \phi \rrbracket_{v}, \llbracket v_{x} . \phi \rrbracket_{v}$ are not defined.

${ }^{3}$ If any of the extremal fixed-points does not exist, then we leave the corresponding expressions undefined.

Manuscript submitted to ACM
} 
Remark 5. We are mainly interested in monotone functions. If $f$ as above is also monotone, then $f$ is compatible if and only if it is strong, meaning that it satisfies

$$
x \wedge f(y) \leq f(x \wedge y), \quad \text { for any } x, y \in H .
$$

The interplay between fixed-points and strong monotone functions has already been emphasized, mainly in the context of categorical proof-theory and semantics of functional programming languages with inductive data types [Clairambault 2013; Cockett and Spencer 1995]. It is well known from categorical literature [Kock 1972] that condition (4) is equivalent to any of the following two conditions:

$$
\begin{aligned}
f(x \rightarrow y) & \leq x \rightarrow f(y), & & \text { for any } x, y \in H, \\
x & \rightarrow y \leq f(x) \rightarrow f(y), & & \text { for any } x, y \in H .
\end{aligned}
$$

Recall that if $v: \mathbb{X}_{\phi} \backslash\{x\} \longrightarrow H$ is a valuation of all the free variables of $\phi$ but $x$, then $(v, h / x): \mathbb{X}_{\phi} \longrightarrow H$ is the valuation such that $(v, h / x)(x)=h$ and $(v, h / x)(y)=v(y)$ for $y \neq x$.

Definition 2. Let $H$ be a Heyting algebra. We say that a function $f: H \longrightarrow H$ is a polynomial if there exist a formula $\phi \in \mathcal{F}_{\text {IPC }}$, a variable $x$, and a valuation $v: \mathbb{X}_{\phi} \backslash\{x\} \longrightarrow H$ such that, for each $h \in H$, we have $f(h)=\llbracket \phi \rrbracket_{(v, h / x)}$.

Equivalently, a polynomial on $H$ can be identified with an element of the polynomial Heyting algebra $H[x]$, where the last is defined as the coproduct (in the category of Heyting algebras) of $H$ with the free Heyting algebra on one generator. In Section 5 we shall study further such polynomial algebras and exploit their properties.

In the next proposition, the analogous statement for Boolean algebras is credited to Peirce, in view of the iteration rule for existential graphs of type Alpha [Dau 2006].

Proposition 6. Every polynomial $f$ on a Heyting algebra is compatible. In particular, if $f$ is monotone, then it is strong.

Proof. Recall that the replacement Lemma holds in the IPC: $\left.z \leftrightarrow w\right|_{\mathbf{L J}} \phi(z) \leftrightarrow \phi(w)$. Substituting $y$ for $z$ and $x \wedge y$ for $w$, and considering that $\left.x\right|_{\mathbf{L J}} y \leftrightarrow(x \wedge y)$, we derive that $\left.x\right|_{\mathbf{L J}} \phi(y) \leftrightarrow \phi(x \wedge y)$. The latter relation is equivalent to the conjunction of $\left.x \wedge \phi(y)\right|_{\mathbf{L J}} x \wedge \phi(x \wedge y)$ and $\left.x \wedge \phi(x \wedge y)\right|_{\mathbf{L J}} x \wedge \phi(y)$. These two relations immediately imply that equation (3) holds when $f$ is a polynomial.

On the way let us include the following Lemma.

Lemma 7. If $f: H \longrightarrow H$ is a strong monotone function and $a \in H$, then

$$
a \rightarrow f(a \rightarrow x)=a \rightarrow f(x) .
$$

Proof. Using (5), we deduce

$$
a \rightarrow f(a \rightarrow x) \leq a \rightarrow(a \rightarrow f(x))=a \rightarrow f(x) .
$$

The converse relation follows from $x \leq a \rightarrow x$ and $a \rightarrow f(x)$ being monotone in $x$.

Proposition 8. If $f$ is a strong monotone function on $H$ and $a \in H$, then

$$
\mu .(a \rightarrow f):=a \rightarrow \mu . f, \quad \quad \mu .(a \wedge f):=a \wedge \mu . f .
$$


Proof. Firstly, we argue that the equation on the left holds. To this end, let us set $f^{a}(x)=_{\text {def }} a \rightarrow f(x)$. From $f \leq f^{a}$ we have $\operatorname{Pre}_{f} a \subseteq \operatorname{Pre}_{f}$. Thus, if $p \in \operatorname{Pre}_{f}$, then $\mu . f=f(\mu . f) \leq f(p)$ and $a \rightarrow \mu . f \leq a \rightarrow f(p)=f^{a}(p) \leq p$. That is, $a \rightarrow \mu$. $f$ is below any element of Pre $_{f} a$. To obtain the proposition, we need to argue that $a \rightarrow \mu$. $f$ belongs to Pre $_{f}$. This follows from equation (7): $f^{a}(a \rightarrow \mu . f)=a \rightarrow f(a \rightarrow \mu . f)=a \rightarrow f(\mu . f)=a \rightarrow \mu . f$.

Let us come now to the equation on the right, for which we set $f_{a}(x)=_{\text {def }} a \wedge f(x)$. Suppose $a \wedge f(p) \leq p$, so $f(p) \leq a \rightarrow p$. Then $f(a \rightarrow p) \leq a \rightarrow f(p) \leq a \rightarrow p$, using (5), whence $\mu . f \leq a \rightarrow p$ and $a \wedge \mu . f \leq p$. Thus we are left to argue that $a \wedge \mu$.f is a prefixed-point of $f_{a}$. Yet, this is true for an arbitrary prefixed-point $p$ of $f$ : $a \wedge f(a \wedge p) \leq a \wedge f(p) \leq a \wedge p$.

CoROllary 9. For each $n \geq 1$ and each collection $f_{i}, i=1, \ldots, n$ of monotone polynomials, we have the following distribution law:

$$
\mu_{x} \cdot \bigwedge_{i=1, \ldots, n} f_{i}(x):=\bigwedge_{i=1, \ldots, n} \mu_{x} \cdot f_{i}(x)
$$

Proof. For $n=1$ there is nothing to prove. We suppose therefore that the statement holds for every collection of size $n \geq 1$, and prove it holds for a collection of size $n+1$. We have

$$
\begin{array}{rlr}
\mu_{x} \cdot\left(f_{n+1}(x) \wedge \bigwedge_{i=1, \ldots, n} f_{i}(x)\right) & :=\mu_{x} \cdot \mu_{y} \cdot\left(f_{n+1}(y) \wedge \bigwedge_{i=1, \ldots, n} f_{i}(x)\right), & \text { by (Diag), } \\
& :=\mu_{x} \cdot\left(\left(\mu_{y} \cdot f_{n+1}(y)\right) \wedge \bigwedge_{i=1, \ldots, n} f_{i}(x)\right), & \text { by (8), } \\
& :=\left(\mu_{y} \cdot f_{n+1}(y)\right) \wedge \mu_{x} \cdot\left(\bigwedge_{i=1, \ldots, n} f_{i}(x)\right), & \text { again by (8), } \\
& :=\left(\mu_{y} \cdot f_{n+1}(y)\right) \wedge \bigwedge_{i=1, \ldots, n} \mu_{x} \cdot f_{i}(x), & \text { by the IH. } \quad \square
\end{array}
$$

The elimination of greatest fixed-points is easy for strong monotone functions. We are thankful to a referee of [Ghilardi et al. 2016] for pointing out the following fact, which greatly simplified our original argument:

Proposition 10. If $f: L \longrightarrow L$ is any strong monotone function on a bounded lattice $L$, then $f^{2}(T)=f(T)$. Thus $f(T)$ is the greatest fixed-point of $f$.

Proof. Indeed, we have $f(T)=f(T) \wedge f(T) \leq f(f(T) \wedge T)=f^{2}(T)$.

\section{BISIMULATION QUANTIFIERS AND FIXED-POINTS}

The connection between extremal fixed-points and bisimulation quantifiers, firstly emphasized in [D'Agostino and Hollenberg 2000], was a main motivation to develop this research. Although in the end the elimination procedure does not rely on it, we nevertheless want to have a closer look at this connection. It was discovered in [Pitts 1992] that IPC has the uniform interpolation property. As it is clear from the title of that work, this property amounts to an internal existential and universal quantification. This result was further refined in [Ghilardi and Zawadowski 1997] to show that any morphism between finitely presented Heyting algebras has a left and a right adjoint.

We shall be interested in Heyting algebras $H[x]$ of polynomials with coefficients in $H$, and in particular mappings from $H[x]$ to $H$, namely the left and right adjoints to the inclusion of $H$ into $H[x]$. The algebra of polynomials $H[x]$ is formally defined as the coproduct (in the category of Heyting algebras) of $H$ with the free Heyting algebra on one generator. The universal property of the coproduct yields that for every $h_{0} \in H$ there exists a unique morphism Manuscript submitted to ACM 
$\llbracket \cdot \rrbracket_{h_{0} / x}: H[x] \longrightarrow H$ such that $\llbracket x \rrbracket_{h_{0} / x}=h_{0}$ and $\llbracket h \rrbracket_{h_{0} / x}=h$, for each $h \in H$. Thus, for $f \in H[x]$ and $h \in H$, we can define the result of evaluating $f$ at $h$ by $f(h)=_{\operatorname{def}} \llbracket f \rrbracket_{h / x}$. If $H$ is finitely generated, then the correspondence sending $h$ to $f(h)=\llbracket f \rrbracket_{h / x}$ is a polynomial on $H$, as defined in Definition 2; moreover, every polynomial in $H$ arises from some $f \in H[x]$ in this way.

An algebra is said to be finitely presented if it is the quotient of a finitely generated free algebra by a finite number of equations, see [Ghilardi and Zawadowski 1997]. In op. cit., it was proved that if $H$ is finitely presented, then the canonical inclusion $i_{x}: H \longrightarrow H[x]$ has both adjoints $\exists_{x}, \forall_{x}: H[x] \longrightarrow H$, with $\exists_{x}$ is left adjoint to $i_{x}$ and $\forall_{x}$ is right adjoint to $i_{x}$. This means that, for each $f \in H[x]$ and $h \in H$, the following equivalences hold:

$$
\exists_{x} \cdot f \leq h \quad \text { iff } \quad f \leq i_{x}(h), \quad h \leq \forall_{x} \cdot f \quad \text { iff } \quad i_{x}(h) \leq f .
$$

From these relations the unit relation for $\exists_{x}$ and the counit relation for $\forall_{x}$ are easily derived:

$$
f \leq i_{x}\left(\exists_{x} . f\right), \quad i_{x}\left(\forall_{x} \cdot f\right) \leq f, \quad \text { for all } f \in H[x] .
$$

We shall use in the rest of this section a standard informal notation: we write $f(x)$ for $f \in H[x]$ and identify $h \in H$ with the constant polynomial $i_{x}(h) \in H[x]$. Using these conventions, the inequalities in (11) are written respectively as $f(x) \leq \exists_{x} . f(x)$ and $\forall_{x} . f(x) \leq f(x)$. We say that $f \in H[x]$ is monotone if the evaluation function it gives rise is monotone, that is, if $\llbracket f \rrbracket_{h_{0} / x} \leq \llbracket f \rrbracket_{h_{1} / x}$ whenever $h_{0} \leq h_{1}$.

Proposition 11. If $f$ is a monotone polynomial on a finitely presented Heyting algebra, then

$$
v \cdot f:=\exists_{x} \cdot(x \wedge(x \rightarrow f(x))) .
$$

Proof. By the unit relation in (11) $x \wedge(x \rightarrow f(x)) \leq \exists_{x} .(x \wedge(x \rightarrow f(x)))$. Recall that evaluation at $p \in H$ is a Heyting algebra morphism, thus it is monotone. Therefore, if $p \in H$ is a postfixed-point of $f$, then by evaluating the previous inequality at $p$, we have

$$
p=p \wedge(p \rightarrow f(p)) \leq \exists_{x} \cdot(x \wedge(x \rightarrow f(x))),
$$

so that $\exists_{x} \cdot(x \wedge(x \rightarrow f(x)))$ is greater than any postfixed-point of $f$. Let us show that $\exists_{x} \cdot(x \wedge(x \rightarrow f(x)))$ is also a postfixed-point. In view of (10) it will be enough to argue that $x \wedge x \rightarrow f(x) \leq f\left(\exists_{x} .(x \wedge x \rightarrow f(x))\right)$ in $H[x]$. We compute as follows:

$$
\begin{aligned}
x \wedge(x \rightarrow f(x)) & \leq f(x) \wedge(x \rightarrow f(x)) \\
& \leq f(x \wedge(x \rightarrow f(x))), \\
& \leq f\left(\exists_{x} .(x \wedge(x \rightarrow f(x)))\right),
\end{aligned}
$$

since $f$ is strong, by (4),

since $f$ is monotone. $\quad \square$

In a similar fashion, we can construct least fixed-points of monotone polynomials using this time universal bisimulation quantifiers.

Proposition 12. If $f$ is a monotone polynomial on a finitely presented Heyting algebra, then

$$
\mu \cdot f:=\forall_{x} \cdot((f(x) \rightarrow x) \rightarrow x) .
$$

Proof. By the counit relation in $(11) \forall_{x} \cdot((f(x) \rightarrow x) \rightarrow x) \leq(f(x) \rightarrow x) \rightarrow x$. Evaluating this relation at $p \in H$ such that $f(p) \leq p$, we obtain

$$
\forall_{x} .((f(x) \rightarrow x) \rightarrow x) \leq(f(p) \rightarrow p) \rightarrow p=\top \rightarrow p=p,
$$


so $\forall_{x} .((f(x) \rightarrow x) \rightarrow x)$ is smaller than any prefixed-point of $f$. We show next that $\forall_{x} \cdot((f(x) \rightarrow x) \rightarrow x)$ is also a prefixed-point of $f$ for which it will be enough to argue that $f(\forall x \cdot((f(x) \rightarrow x) \rightarrow x)) \leq(f(x) \rightarrow x) \rightarrow x$ in $H[x]$ or, equivalently, that $(f(x) \rightarrow x) \wedge f\left(\forall_{x} .((f(x) \rightarrow x) \rightarrow x)\right) \leq x$. We compute as follows:

$$
\begin{aligned}
(f(x) \rightarrow x) \wedge f\left(\forall_{x} \cdot((f(x) \rightarrow x) \rightarrow x)\right) & =(f(x) \rightarrow x) \wedge f\left((f(x) \rightarrow x) \wedge \forall_{x} \cdot((f(x) \rightarrow x) \rightarrow x)\right), \\
\text { where we use that } f \text { is strong, } & \leq(f(x) \rightarrow x) \wedge f(x) \leq x
\end{aligned}
$$

where in the last inequality we have used that $f$ is monotone and the inequality $(f(x) \rightarrow x) \wedge \forall_{x} \cdot((f(x) \rightarrow x) \rightarrow x) \leq x$, equivalent to the counit relation for $(f(x) \rightarrow x) \rightarrow x$.

The next result is an immediate consequence of Propositions 6 and 10. However the previous proposition yields now an alternative proof:

COROLLARY 13. If $f$ is a monotone polynomial on a finitely presented Heyting algebra $H$, then

$$
v \cdot f:=f(T) .
$$

Proof. It is easy to see that if $f$ is a monotone polynomial on a finitely presented Heyting algebra, then $\exists_{x} \cdot f=f(\top)$. Thus we have

$$
v \cdot f=\exists_{x} \cdot(x \wedge(x \rightarrow f(x)))=\exists_{x} \cdot(x \wedge f(x))=\top \wedge f(\top)=f(\top) .
$$

Let us come back to a more syntactic perspective. If $\phi(x)$ is a formula-term positive in $x$ whose variables distinct from $x$ are among $y_{1}, \ldots, y_{n}$, then the equality $\phi^{2}(T)=\phi(T)$ holds in the free Heyting algebra on the set $\left\{y_{1}, \ldots, y_{n}\right\}$ (which is finitely presented). Since such a free Heyting algebra is a subalgebra of the Lindenbaum-Tarski algebra, this means that $\left.\phi(T)\right|_{\mathbf{L J}} \phi(\phi(T))$ and $\left.\phi(\phi(T))\right|_{\mathbf{L J}} \phi(T)$. A similar argument, using free Heyting algebras, can be used to generalize Corollary 13 to all Heyting algebras, thus leaving out the requirement that the algebra $H$ is finitely presented.

\section{THE ELIMINATION PROCEDURE}

We present in this Section our first main result, a procedure that both axiomatizes and eliminates least fixed-points of the form $\mu_{x} . \phi$ with $\phi$ fixed-point free. Together with the axiomatization of greatest fixed-points given in Proposition 10 and Corollary 13, the procedure can be extended to a procedure to construct a fixed-point free formula $\psi$ equivalent to a given formula $\chi$ of the $\operatorname{IPC}_{\mu}$. To ease the reading of the content of this Section and of the remaining ones, we introduce the following notation:

$$
[\alpha] \phi=_{\text {def }} \alpha \rightarrow \phi
$$

When using the notation above, we shall always assume that the special variable $x$ does not occur in the formula $\alpha$.

Definition 3. An occurrence of the variable $x$ is strongly positive in a formula-term $\phi$ if there is no subformula $\psi$ of $\phi$ of the form $\psi_{0} \rightarrow \psi_{1}$ such that $x$ is located in $\psi_{0}$. A formula-term $\phi$ is strongly positive in the variable $x$ if every occurrence of $x$ is strongly positive in $\phi$. An occurrence of a variable $x$ is weakly negative in a formula-term $\phi$ if it is not strongly positive. A formula-term $\phi$ is weakly negative in the variable $x$ if every occurrence of $x$ is weakly negative in $\phi$.

We shall also say that a variable $x$ is strongly positive (resp. weakly negative) in a formula $\phi$ when $\phi$ is strongly positive (resp. weakly negative) in the variable $x$. Observe that a variable might be neither strongly positive nor weakly negative in a formula-term.

Manuscript submitted to ACM 


\subsection{Summary of the procedure}

In order to compute the least fixed-point $\mu_{x} . \phi$, we take the following steps:

(1) We rename all the weakly negative occurrences of $x$ in $\phi$ to a fresh variable $y$, so $\phi(x)=\psi(x, x / y)$ with $\psi$ strongly positive in $x$ and weakly negative in $y$.

(2) Computation of a normal form. We compute a normal form of $\psi(x, y)$, that is, a formula equivalent to $\psi(x, y)$ which is a conjunction $\bigwedge_{i \in I} \psi_{i}(x, y)$ with each $\psi_{i}$ disjunctive in $x$ (see Definition 4 below) or not containing the variable $x$.

(3) Strongly positive elimination. For each $i \in I$ : if $x$ has an occurrence in $\psi_{i}$, we compute then a formula $\psi_{i}^{\prime}$ equivalent to the least fixed-point $\mu_{x} \cdot \psi_{i}(x, y)$ and observe that $\psi_{i}^{\prime}$ is weakly negative in $y$; otherwise, we let $\psi_{i}^{\prime}=\psi_{i}$.

(4) Weakly negative elimination. The formula $\bigwedge_{i \in I} \psi_{i}^{\prime}(y)$ is weakly negative in $y$; we compute a formula $\chi$ equivalent to $\mu_{y} \cdot \wedge_{i} \psi_{i}^{\prime}(y)$ and return it.

The correctness of the procedure relies on the following chain of equivalences:

$$
\begin{aligned}
\mu_{x} \cdot \phi(x) & =\mu_{y} \cdot \mu_{x} \cdot \psi(x, y), \\
& =\mu_{y} \cdot \mu_{x} \cdot \bigwedge_{i \in I} \psi_{i}(x, y)=\mu_{y} \cdot \bigwedge_{i \in I} \mu_{x} \cdot \psi_{i}(x, y), \\
& =\mu_{y} \cdot \bigwedge_{i \in I} \psi_{i}^{\prime}(y)=\chi .
\end{aligned}
$$

where we use (Diag), using Corollary 9 ,

\subsection{Computation of a normal form}

If a formula-term $\phi$ does not contain the variable $x$, then $x$ is both strongly positive and weakly negative in this formula. Yet, in this case, we have $\mu_{x} . \phi=\phi$, thus it is a trivial case for the sake of computing its least fixed-point. For this reason we present below a grammar recognising strongly positive formula-terms containing the variable $x$. The grammar is

$$
\phi \Rightarrow x|[\alpha] \phi| \beta \vee \phi|\phi \vee \phi| \phi \wedge \phi \mid \gamma \wedge \phi
$$

where conjunctions and disjunctions are taken up to commutativity and where $\alpha, \beta, \gamma$ do not contain the variable $x$. Another key concept for the elimination procedure is the notion of disjunctive formula, obtained by eliminating the last two productions from the above grammar.

Definition 4. The set of formula-terms that are disjunctive in the variable $x$ is generated by the grammar

$$
\phi \Rightarrow x|[\alpha] \phi| \beta \vee \phi \mid \phi \vee \phi
$$

where $\alpha$ and $\beta$ are formulas with no occurrence of the variable $x$. A formula-term $\phi$ is in normal form (w.r.t. $x$ ) if it is a conjunction of formula-terms $\phi_{i}, i \in I$, so that each $\phi_{i}$ either does not contain the variable $x$, or it is disjunctive in $x$.

Due to equation (2) and since the usual distributive laws hold in Heyting algebras, every strongly positive formulaterm is equivalent to a formula-term in normal form, as witnessed by the following Lemma.

LEMMA 14. Every formula-term that is strongly positive in $x$ and contains the variable $x$ is equivalent to a conjunction of disjunctive formulas and of a formula that does not contain $x$.

Proof. By induction, we associate to each such formula a set $\operatorname{tr}(\phi)$ of disjunctive formulas and formula $c(\phi)$ so that

$$
\phi={ }_{\mathrm{IPC}} c(\phi) \wedge \bigwedge\{\delta \mid \delta \in \operatorname{tr}(\phi)\} .
$$


We let

$$
\begin{aligned}
& \operatorname{tr}(x)={ }_{\text {def }}\{x\}, \\
& \operatorname{tr}([\alpha] \phi)==_{\operatorname{def}}\{[\alpha] \delta \mid \delta \in \operatorname{tr}(\phi)\}, \\
& c(x)==_{\text {def }} \top \text {; } \\
& \operatorname{tr}(\beta \vee \phi)=_{\operatorname{def}}\{\beta \vee \delta \mid \delta \in \operatorname{tr}(\phi)\}, \\
& c([\alpha] \phi)={ }_{\operatorname{def}}[\alpha] c(\phi) ; \\
& \operatorname{tr}\left(\phi_{1} \vee \phi_{2}\right)=_{\text {def }}\left\{c\left(\phi_{1}\right) \vee \delta_{2} \mid \delta_{2} \in \operatorname{tr}\left(\phi_{2}\right)\right\} \\
& c(\beta \vee \phi)={ }_{\operatorname{def}} \beta \vee c(\phi) ; \\
& \cup\left\{c\left(\phi_{2}\right) \vee \delta_{1} \mid \delta_{1} \in \operatorname{tr}\left(\phi_{1}\right)\right\} \\
& \cup\left\{\delta_{1} \vee \delta_{2} \mid \delta_{1} \in \operatorname{tr}\left(\phi_{1}\right), \delta_{2} \in \operatorname{tr}\left(\phi_{2}\right)\right\}, \\
& \operatorname{tr}\left(\phi_{1} \wedge \phi_{2}\right)={ }_{\text {def }} \operatorname{tr}\left(\phi_{1}\right) \cup \operatorname{tr}\left(\phi_{2}\right), \\
& c\left(\phi_{1} \wedge \phi_{2}\right)=\mathrm{def}_{\mathrm{def}} c\left(\phi_{1}\right) \wedge c\left(\phi_{2}\right) ; \\
& \operatorname{tr}(\gamma \wedge \phi)=_{\text {def }} \operatorname{tr}(\phi), \\
& c(\gamma \wedge \phi)=\operatorname{def} \gamma \wedge c(\phi) .
\end{aligned}
$$

Verification that (16) holds is routine.

\subsection{Strongly positive elimination}

We tackle here the problem of computing the least fixed-point $\mu_{x} . \phi$ of a formula-term $\phi$ which is disjunctive in $x$. Recall that the formulas $\alpha$ and $\beta$ appearing in a parse tree as leaves-according to the grammar (15)-do not contain the variable $x$. We call such a formula $\alpha$ a head subformula of $\phi$, and such a $\beta$ a side subformula of $\phi$, and thus we put:

$$
\operatorname{Head}(\phi)=_{\text {def }}\{\alpha \mid \alpha \text { is a head subformula of } \phi\}, \quad \operatorname{Side}(\phi)=_{\operatorname{def}}\{\beta \mid \beta \text { is a side subformula of } \phi\} .
$$

Recall that a monotone function $f: P \longrightarrow P$ is inflating if $x \leq f(x)$, for all $x \in P$.

LEMMA 15. The interpretation of a strongly positive disjunctive formula $\phi$ as a function of $x$ is inflating.

The proof of this lemma is by induction, using the grammar (15) that defines disjunctive formulas.

The key observation needed to prove Proposition 17 below is the following Lemma on monotone inflating functions. In the statement of the lemma we assume that $P$ is a join-semilattice, and that $f \vee g$ is the pointwise join of the two functions $f$ and $g$.

Lemma 16. If $f, g: P \longrightarrow P$ are monotone inflating functions, then $f \vee g$ and $f \circ g$ are monotone inflating and Pre $_{f \vee g}=\operatorname{Pre}_{f \circ g}$. Consequently, for any monotone function $h: P \longrightarrow P$, we have

$$
\mu .(f \vee g \vee h):=: \mu .((f \circ g) \vee h) .
$$

Proof. It is easy to see that $f \vee g$ and $f \circ g$ are monotone inflating, so we only verify that $\operatorname{Pre}_{f \vee g}=\operatorname{Pre}_{f \circ g}$. Observe firstly that $\operatorname{Pre}_{f \vee g}=\operatorname{Pre}_{f} \cap \operatorname{Pre}_{g}$. If $p \in \operatorname{Pre}_{f \circ g}$, then $f(p) \leq f(g(p)) \leq p$ and $g(p) \leq f(g(p)) \leq p$, showing that $p \in \operatorname{Pre}_{f \vee g}$. Conversely, if $p \in \operatorname{Pre}_{f \vee g}$, then $p$ is a fixed point of both $f$ and $g$, since these functions are inflating. It follows that $f(g(p))=f(p)=p$, showing $p \in$ Pre $_{f \circ g}$.

We have argued that $\operatorname{Pre}_{f \vee g}$ coincides with $\operatorname{Pre}_{f \circ g}$; this implies that $\operatorname{Pre}(f \circ g) \vee h=\operatorname{Pre}_{f \vee g \vee h}$ and, from this equality, equation (17) immediately follows.

Proposition 17. If $\phi$ is a disjunctive formula-term, then

$$
\mu_{x} \cdot \phi:=\left[\bigwedge_{\alpha \in \operatorname{Head}(\phi)} \alpha\right]\left(\bigvee_{\beta \in \operatorname{Side}(\phi)} \beta\right) .
$$

Manuscript submitted to ACM 
Proof. For $\psi, \chi$ formula-terms, let us write $\psi \sim \chi$ when $\mu_{x} \cdot \psi=\mu_{x} \cdot \chi$. We say that a disjunctive formula $\psi$ is reduced (w.r.t. $\phi$ ) if either it is $x$, or it is of the form $\beta \vee x$ (or $x \vee \beta$ ) for some $\beta \in \operatorname{Side}(\phi)$ ), or of the form $[\alpha] x$ for some $\alpha \in \operatorname{Head}(\phi)$. A set $\Phi$ of disjunctive formulas is reduced if every formula in $\Phi$ is reduced.

We shall compute a reduced set of disjunctive formulas $\Phi_{k}$ such that $\phi \sim \vee \Phi_{k}$. Thus let $\Phi_{0}=\{\phi\}$. If $\Phi_{i}$ is not reduced, then there is $\phi_{0} \in \Phi_{i}$ which is not reduced, thus of the form (a) $\beta \vee \psi$ (or $\psi \vee \beta$ ) with $\psi \neq x$, or (b) $[\alpha] \psi$ with $\psi \neq x$, or $(\mathrm{c}) \psi_{1} \vee \psi_{2}$. According to the case $(\ell)$, with $\ell \in\{a, b, c\}$, we let $\Phi_{i+1}$ be $\left(\Phi_{i} \backslash\left\{\phi_{0}\right\}\right) \cup \Psi_{\ell}$ where $\Psi_{\ell}$ is as follows:

$$
\Psi_{a}=\{\beta \vee x, \psi\}, \quad \Psi_{b}=\{[\alpha] x, \psi\}, \quad \Psi_{c}=\left\{\psi_{1}, \psi_{2}\right\} .
$$

By Lemma 16, we have $\bigvee \Phi_{i} \sim \bigvee \Phi_{i+1}$. Moreover, for some $k \geq 0, \Phi_{k}$ is reduced and $\Phi_{k} \subseteq\{[\alpha] x \mid \alpha \in \operatorname{Head}(\phi)\} \cup$ $\{\beta \vee x \mid \beta \in \operatorname{Side}(\phi)\} \cup\{x\}$. Consequently

$$
\mu_{x} \cdot \phi(x)=\mu_{x} \cdot \bigvee \Phi_{k} \leq \mu_{x} \cdot\left(x \vee \bigvee_{\alpha \in \operatorname{Head}(\phi)}[\alpha] x \vee \bigvee_{\beta \in \operatorname{Side}(\phi)} \beta \vee x\right) .
$$

On the other hand, if $\alpha \in \operatorname{Head}(\phi)$, then $\phi(x)=\psi_{1}\left(x,[\alpha] \psi_{2}(x)\right)$ for some disjunctive formulas $\psi_{1}$ and $\psi_{2}$, so

$$
[\alpha] x \leq[\alpha] \psi_{2}(x) \leq \psi_{1}\left(x,[\alpha] \psi_{2}(x)\right)=\phi(x)
$$

and, similarly, $\beta \vee x \leq \phi(x)$, whenever $\beta \in \operatorname{Side}(\phi)$. It follows that

$$
x \vee \bigvee_{\alpha \in \operatorname{Head}(\phi)}[\alpha] x \vee \bigvee_{\beta \in \operatorname{Side}(\phi)} \beta \vee x \leq \phi(x),
$$

whence, by taking the least fixed-point in both sides of the above inequality, we derive equality in (19). Finally, in order to obtain (18), we compute as follows:

$$
\begin{aligned}
& \mu_{x} .\left(x \vee \vee_{\alpha \in \operatorname{Head}(\phi)}[\alpha] x \vee \vee_{\beta \in \operatorname{Side}(\phi)} \beta \vee x\right) \\
& =\mu_{x} \cdot\left(\left[\alpha_{1}\right] \ldots\left[\alpha_{n}\right] x \vee\left(x \vee \bigvee_{\beta \in \operatorname{Side}(\phi)} \beta \vee x\right)\right) \quad \text { by Lemma 16, with } \operatorname{Head}(\phi)=\left\{\alpha_{1}, \ldots, \alpha_{n}\right\}, \\
& \left.=\mu_{x} \cdot\left(\bigwedge_{\alpha \in \operatorname{Head}(\phi)} \alpha\right] x \vee\left(x \vee \bigvee_{\beta \in \operatorname{Side}(\phi)} \beta \vee x\right)\right), \quad \text { since }\left[\alpha_{1}\right] \ldots\left[\alpha_{n}\right] x=\left[\bigwedge_{i=1, \ldots, n} \alpha_{i}\right] x \\
& =\mu_{x} \cdot\left(\left[\bigwedge_{\alpha \in \operatorname{Head}(\phi)} \alpha\right]\left(x \vee \bigvee_{\beta \in \operatorname{Side}(\phi)} \beta \vee x\right)\right), \quad \text { by Lemma 16, } \\
& =\left[\bigwedge_{\alpha \in \operatorname{Head}(\phi)} \alpha\right] \mu_{x} \cdot\left(x \vee \bigvee_{\beta \in \operatorname{Side}(\phi)} \beta \vee x\right), \quad \text { by Proposition 8, } \\
& =\left[\bigwedge_{\alpha \in \operatorname{Head}(\phi)} \alpha\right]\left(\bigvee_{\beta \in \operatorname{Side}(\phi)} \beta\right) \text {. }
\end{aligned}
$$

Example 18. Formula (18) yields

$$
\mu_{x} \cdot\left(\left[\alpha_{1}\right]\left(\beta_{1} \vee x\right) \vee\left[\alpha_{2}\right]\left(\beta_{2} \vee x\right)\right)=\left[\alpha_{1} \wedge \alpha_{2}\right]\left(\beta_{1} \vee \beta_{2}\right)
$$

Remark 19. Let $\phi$ be a disjunctive formula and consider an occurrence in $\phi$ of a variable $y$ distinct from $x$. Necessarily, such an occurrence is located in some head subformula or in some side subformula of $\phi$. Therefore we can map such an occurrence to an occurrence of the same variable within the formula on the right of the equality (18); notice that a 
weakly negative occurrence is mapped to a weakly negative occurrence. Since every occurrence of a variable $y$ in the formula on the right of (18) has a preimage through the mapping, we conclude the following observation, which is necessary for the global elimination procedure to work: if a variable $y$ is weakly negative in the disjunctive formula $\phi$, then it is still weakly negative in the formula $\mu_{x} . \phi$ as defined by equation (18). Similarly, if $\phi$ is strongly positive in $x$ and weakly negative in $y$, then $y$ is weakly negative in each conjunct appearing on the right of equation (16).

\subsection{Weakly negative elimination}

Recall that we are considering formulas $\phi$ in which every occurrence of the variable $x$ is positive. Therefore, if $\phi$ is weakly negative in $x$, then we can write

$$
\phi(x)=\psi_{0}\left(\psi_{1}(x), \ldots, \psi_{n}(x)\right)
$$

for formula-terms $\psi_{0}\left(y_{1}, \ldots, y_{n}\right)$ and $\psi_{i}(x), i=1, \ldots, n$, such that: (a) all the variables $y_{i}$ are negative in $\psi_{0}$; (b) for $i=1, \ldots, n, x$ is negative $\psi_{i}$.

Proposition 20. Let $\phi$ be a formula which is weakly negative in $x$. Let $\left\langle v_{1}, \ldots, v_{n}\right\rangle$ be a collection of formula-terms denoting the greatest solution of the system of equations $\left\{y_{i}=\psi_{i}\left(\psi_{0}\left(y_{1}, \ldots, y_{n}\right)\right) \mid i=1, \ldots, n\right\}$. Then $\psi_{0}\left(v_{1}, \ldots, v_{n}\right)$ is a formula equivalent to $\mu_{x} . \phi(x)$.

Proof. Let $v: \mathbb{X} \backslash\left\{x, y_{1}, \ldots, y_{n}\right\} \longrightarrow H$ be a partial valuation into a Heyting algebra $H$, put $f_{0}=\llbracket \psi_{0} \rrbracket v$ and, for $i=1, \ldots, n, f_{i}=\llbracket \psi_{i} \rrbracket_{v}$. Then $f_{0}$ is a monotone function from $\left[H^{o p}\right]^{n}$ to $H$. Here $H^{o p}$ is the poset with the same elements as $H$ but with the opposite ordering relation. Similarly, for $1 \leq i \leq n, f_{i}: H \longrightarrow H^{o p}$. If we let $\bar{f}=\left\langle f_{i} \mid i=1, \ldots, n\right\rangle \circ f_{0}$, then $\bar{f}:\left[H^{o p}\right]^{n} \longrightarrow\left[H^{o p}\right]^{n}$. We exploit next the fact that $(\cdot)^{o p}$ is a functor, so that $f^{o p}: P^{o p} \longrightarrow Q^{o p}$ is the same monotone function as $f$, but considered as having distinct domain and codomain. Then, using (Roll), we can write

$$
\mu \cdot\left(f_{0} \circ\left\langle f_{i} \mid i=1, \ldots, n\right\rangle\right)=f_{0}\left(\mu \cdot\left(\left\langle f_{i} \mid i=1, \ldots, n\right\rangle \circ f_{0}\right)\right)=f_{0}(\mu \cdot \bar{f})=f_{0}\left(v \cdot \bar{f}^{o p}\right),
$$

since the least fixed-point of $f$ in $P^{o p}$ is the greatest fixed-point of $f^{o p}$ in $P$. That is, if we consider the function $\left\langle f_{i} \mid i=1, \ldots, n\right\rangle \circ f_{0}$ as sending a tuple of elements of $H$ (as opposite to $H^{o p}$ ) to another such a tuple, then equation (21) proves that a formula denoting the least fixed-point of $\phi$ is constructible out of formulas for the greatest solution of the system mentioned in the statement of the proposition.

As far as computing the greatest solution of the system mentioned in the proposition, this can be achieved by using the Bekic elimination principle (see Lemma 3). This principle implies that solutions of systems can be constructed from solutions of linear systems, i.e. from usual parametrized fixed-points. In our case, as witnessed by equation (13), these parametrized greatest fixed-points are computed by substituting $T$ for the fixed-point variable.

Example 21. Consider the weakly negative $\phi$ defined by

$$
\phi(x)==_{\operatorname{def}}((x \rightarrow c) \rightarrow a) \vee((x \rightarrow d) \rightarrow b) .
$$

We can take then

$$
\psi_{0}\left(y_{1}, y_{2}\right)=_{\text {def }} y_{1} \rightarrow a \vee y_{2} \rightarrow b, \quad \psi_{1}(x)={ }_{\text {def }} x \rightarrow c, \quad \psi_{2}(x)=x \rightarrow d .
$$

The system of equations whose greatest solution we need to compute is

$$
y_{1}=\psi_{1}\left(\psi_{0}\left(y_{1}, y_{2}\right)\right)=\left(y_{1} \rightarrow a \vee y_{2} \rightarrow b\right) \rightarrow c, \quad y_{2}=\psi_{2}\left(\psi_{0}\left(y_{1}, y_{2}\right)\right)=\left(y_{1} \rightarrow a \vee y_{2} \rightarrow b\right) \rightarrow d
$$


The Bekic elimination principle is used to find this solution:

$$
\begin{aligned}
v_{y_{2}} \cdot \psi_{2}\left(\psi_{0}\left(y_{1}, y_{2}\right)\right) & =v_{y_{2}} \cdot\left(\left(y_{1} \rightarrow a\right) \vee\left(y_{2} \rightarrow b\right)\right) \rightarrow d=\left(\left(y_{1} \rightarrow a\right) \vee(\top \rightarrow b)\right) \rightarrow d=\left(\left(y_{1} \rightarrow a\right) \vee b\right) \rightarrow d, \\
v_{1} & =v_{y_{1}} \cdot \psi_{1}\left(\psi_{0}\left(y_{1}, v_{y_{2}} \cdot \psi_{2}\left(\psi_{0}\left(y_{1}, y_{2}\right)\right)\right)\right)=\psi_{1}\left(\psi_{0}\left(\top, v_{y_{2}} \cdot \psi_{2}\left(\psi_{0}\left(\top, y_{2}\right)\right)\right)\right) \\
& =(\top \rightarrow a \vee(((\top \rightarrow a) \vee b) \rightarrow d) \rightarrow b) \rightarrow c=(a \vee(((a \vee b) \rightarrow d) \rightarrow b)) \rightarrow c, \\
v_{2} & =\left(\left(v_{1} \rightarrow a\right) \vee b\right) \rightarrow d .
\end{aligned}
$$

Then, by (Roll), we have $\mu_{x} . \phi(x)=v_{1} \rightarrow a \vee v_{2} \rightarrow b$.

In the next Section, Proposition 29 shall provide an alternative of the least fixed-point of a weakly negative formula $\phi$ by means of approximants.

\section{UPPER BOUNDS FOR CLOSURE ORDINALS}

The closure ordinal of $\phi(x) \in \mathcal{F}_{\text {IPC }}$ is the least integer $n$ for which we can write $\mu_{x} \cdot \phi(x)=\phi^{n}(\perp)$. In view of the proof of Proposition 4, the closure ordinal always exists, for each intuitionistic formula $\phi(x)$ positive on $x$. Closure ordinals yield a representation of least fixed-points of formulas alternative to the one presented in the previous Section. Such representation can be exploited notationally, as in $\mu$-calculi with explicit approximations [Dam and Gurov 2002]. Also it can be exploited computationally because of the reduced space requirements, at least when variable sharing is used. Finally, it can be exploited to provide axiomatizations. In this Section we begin the study of (finite) closure ordinals.

\subsection{General results}

In this Section all the posets we consider shall have a least element, denoted by $\perp$ as usual. We say that a monotone function $f: P \longrightarrow P$ converges in $n$ steps to its least fixed-point if $f^{n+1}(\perp)=f^{n}(\perp)$ or, equivalently, if $\mu . f=f^{n}(\perp)$; in such a case the least of those integers $n$ is called the closure ordinal of $f$ and it is denoted by $\operatorname{cl}(f)$. We informally call the $f^{n}(\perp), n \geq 0$, the approximants (or approximations) of (the least fixed-point of) $f$. If $f: Q \times P^{n} \longrightarrow P^{k}$ is a monotone function and $\left\{i_{1}<i_{2}<\ldots<i_{k}\right\} \subseteq\{1, \ldots, n\}$, then we write $\operatorname{cl}_{\left(x_{i_{1}}, \ldots, x_{i_{k}}\right)}(f) \leq n$ if, for each $q \in Q$ and $\vec{p} \in P^{n-k}, \operatorname{cl}\left(f_{(q, \vec{p})}\right) \leq n$, where $f_{(q, \vec{p})}: P^{k} \longrightarrow P^{k}$ is the monotone function obtained from $f$ by fixing $q \in Q$ and evaluating all the variables $x_{j}$ with $j \notin\left\{i_{1}, \ldots, i_{k}\right\}$ by means of the vector $\vec{p}$.

The next propositions suggest how to compute convergence of monotone functions based on the properties of least fixed-points that we have introduced in Section 2.

Proposition 22 (Convergence for (Roll)). Let $f: P \longrightarrow Q$ and $g: Q \longrightarrow P$ be monotone functions. If $\mu .(f \circ g)=$ $(f \circ g)^{n}(\perp)$, then $\mu .(g \circ f)=(g \circ f)^{n+1}(\perp)$. Therefore $\operatorname{cl}(g \circ f) \leq 1+\operatorname{cl}(f \circ g)$.

Proof. We observe that

$$
\mu .(g \circ f)=g(\mu .(f \circ g))=g \circ(f \circ g)^{n}(\perp) \leq g \circ(f \circ g)^{n}(f(\perp))=(g \circ f)^{n+1}(\perp) .
$$

Since the converse inclusion always holds, we have proved the proposition.

Example 23. Consider $\phi(x)=_{\mathrm{def}}(x \rightarrow b) \rightarrow a$. By using Proposition 20 (with $\psi_{0}\left(y_{1}\right)=_{\mathrm{def}}\left(y_{1} \rightarrow a\right)$ and $\psi_{1}(x)=_{\mathrm{def}}$ $(x \rightarrow b))$ we know that

$$
\mu_{x} \cdot \phi(x)=\left(v_{x} \cdot(x \rightarrow a) \rightarrow b\right) \rightarrow a=((\top \rightarrow a) \rightarrow b) \rightarrow a=(a \rightarrow b) \rightarrow a
$$

Manuscript submitted to ACM 
Otherwise, we can combine Propositions 10 and 22 to deduce $\mu_{x} \cdot \phi(x)=\phi^{2}(\perp)$. Indeed, a direct computation of the approximants yields

$$
\phi(\perp)=a, \phi^{2}(\perp)=(a \rightarrow b) \rightarrow a .
$$

This example shows that the bound on the convergence given in Proposition 22 is tight, since the equality $\phi^{2}(\perp)=\phi(\perp)$ only holds for arbitrary $a$ and $b$ whenever $H$ is a Boolean algebra. As a matter of fact, note that this equality is Peirce's law

$$
(a \rightarrow b) \rightarrow a=a,
$$

which forces a Heyting algebra to be Boolean.

Proposition 24 (Convergence for (Bekic)). Let $\langle f, g\rangle: P \times Q \longrightarrow P \times Q$ be a monotone mapping. Put $h(x)={ }_{\mathrm{def}}$ $f\left(x, \mu_{y} \cdot g(x, y)\right)$. Let $m, n \geq 0$ be such that $\mu_{y} \cdot g(x, y)=g_{x}^{m}(\perp)$ for each $x \in P$ and $\mu_{x} \cdot h(x)=h^{n}(\perp)$. Then

$$
\mu .\langle f, g\rangle=\langle f, g\rangle^{(n+1)(m+1)-1}(\perp, \perp) .
$$

That is, $\operatorname{cl}(\langle f, g\rangle) \leq\left(\mathrm{cl}_{y}(g)+1\right)(\mathrm{cl}(h)+1)-1$.

Proof. Let us define by induction the following sequences:

$$
\begin{aligned}
\mathfrak{f}_{0}=\mathfrak{g}_{0}=\perp, & \mathfrak{f}_{i+1}=f\left(\mathfrak{f}_{i}, \mathfrak{g}_{i}\right), & \mathfrak{g}_{i+1}=g\left(\tilde{f}_{i}, \mathfrak{g}_{i}\right), \\
\kappa_{0}=\mathfrak{h}_{0}=\perp, & \kappa_{i+1}=\left(g_{\mathfrak{h}_{i}}\right)^{m}(\perp), & \mathfrak{h}_{i+1}=f\left(\mathfrak{h}_{i}, \kappa_{i+1}\right) .
\end{aligned}
$$

Notice first that, for each $i \geq 0,\langle f, g\rangle^{i}(\perp, \perp)=\left\langle\tilde{f}_{i}, \mathfrak{g}_{i}\right\rangle$, On the other hand, we have

$$
\mathfrak{h}_{i+1}=f\left(\mathfrak{h}_{i}, \kappa_{i+1}\right)=f\left(\mathfrak{h}_{i},\left(g_{\mathfrak{h}_{i}}\right)^{m}(\perp)\right)=f\left(\mathfrak{h}_{i}, \mu_{y} \cdot g\left(\mathfrak{h}_{i}, y\right)\right)=h\left(\mathfrak{h}_{i}\right),
$$

so, by a straightforward induction, we obtain that $\mathfrak{h}_{i}=h^{i}(\perp)$. Then, by the Bekic property,

$$
\mu .\langle f, g\rangle=\left\langle h^{n}(\perp),\left(g_{h^{n}(\perp)}\right)^{m}(\perp)\right\rangle=\left\langle\mathfrak{h}_{n}, \kappa_{n+1}\right\rangle .
$$

Claim. Let $\psi: \mathbb{N} \longrightarrow \mathbb{N}$ be any function. For each $i \geq 0$,

(1) $\mathfrak{h}_{i} \leq \mathfrak{f}_{\psi(i)}$ implies $\kappa_{i+1} \leq \mathfrak{g}_{\psi(i)+m}$

(2) $\mathfrak{h}_{i} \leq \mathfrak{f}_{\psi(i)}$ implies $\mathfrak{h}_{i} \leq \mathfrak{f}_{\psi(i)+m+1}$.

Proof of Claim. (1) Let us suppose that $\mathfrak{h}_{i} \leq \mathfrak{f}_{\psi(i)}$ and prove that $\left(g_{\mathfrak{h}_{i}}\right)^{\ell}(\perp) \leq \mathfrak{g}_{\psi(i)+\ell}$ for $\ell=0, \ldots, m$. This relation trivially holds for $\ell=0$ and, supposing it holds for $\ell$,

$$
\begin{aligned}
\left(g_{\mathfrak{h}_{i}}\right)^{\ell+1}(\perp) & =g_{\mathfrak{h}_{i}}\left(g_{\mathfrak{h}_{i}}^{\ell}(\perp)\right) \leq g_{\mathfrak{h}_{i}}\left(\mathfrak{g}_{\psi(i)+\ell}\right)=g\left(\mathfrak{h}_{i}, \mathfrak{g}_{\psi(i)+\ell}\right) \\
& \leq g\left(\mathfrak{\mathfrak { f }}_{\psi(i)}, \mathfrak{g}_{\psi(i)+\ell}\right) \leq g\left(\mathfrak{f}_{\psi(i)+\ell}, \mathfrak{g}_{\psi(i)+\ell}\right)=\mathfrak{g}_{\psi(i)+\ell+1} .
\end{aligned}
$$

Thus, for $\ell=m$, we have $\kappa_{i+1}=\left(g_{\mathfrak{h}_{i}}\right)^{m}(\perp) \leq \mathfrak{g}_{\psi(i)+m}$.

(2) If we suppose $\mathfrak{h}_{i} \leq \mathfrak{f}_{\psi(i)}$, then $\kappa_{i+1} \leq \mathfrak{g}_{\psi(i)+m}$ by (1), and

$$
\mathfrak{h}_{i+1}=f\left(\mathfrak{h}_{i}, \kappa_{i+1}\right) \leq f\left(\mathfrak{f}_{\psi(i)}, \mathfrak{g}_{\psi(i)+m}\right) \leq f\left(\mathfrak{f}_{\psi(i)+m}, \mathfrak{g}_{\psi(i)+m}\right)=\mathfrak{f}_{\psi(i)+m+1} .
$$


If now we let $\psi(i)==_{\text {def }} i(m+1)$, then $\mathfrak{h}_{i} \leq \mathfrak{f}_{\psi(i)}$, for all $i \geq 0$, by induction on $i$ and using part (2) of the Claim. Then we deduce that

$$
\begin{aligned}
\mu .\langle f, g\rangle & =\left\langle\mathfrak{h}_{n}, \kappa_{n+1}\right\rangle \\
& \leq\left\langle\mathfrak{f}_{\psi(n)}, \mathfrak{g}_{\psi(n)+m}\right\rangle \leq\left\langle\mathfrak{f}_{\psi(n)+m}, \mathfrak{g}_{\psi(n)+m}\right\rangle=\langle f, g\rangle^{\psi(n)+m}(\perp, \perp),
\end{aligned}
$$

showing that the function $\langle f, g\rangle$ converges to its least fixed-point in $\psi(n)+m=(n+1)(m+1)-1$ steps.

Example 25. We argue that the upper bound $(n+1)(m+1)-1=(m+1) n+m$ given in Proposition 24 is tight. For $m, n \geq 1$, let $P$ and $Q$ be respectively the $n+1$-element chain $\{0<1<\ldots<n\}$ and the $(n+1) m+1$-element chain $\{0<1<\ldots<(n+1) m\}$. On these chains define the successor function $s$ by $s(x)=x+1$ if $x \neq \top$ and, otherwise, $s(T)=\mathrm{T}$. If $y \in Q$, then it can be written in the form $z m+k$ for some $0 \leq k<m$ and $0 \leq z \leq n+1$. Define the mappings $f: P \times Q \rightarrow P$ and $g: P \times Q \rightarrow Q$ as follows:

$$
\begin{aligned}
& f(x, z m+k)= \begin{cases}x, & \text { if } z \leq x, \\
s(x), & \text { otherwise, }\end{cases} \\
& g(x, z m+k)= \begin{cases}x m+k+1, & \text { if } z \leq x, \\
(x+1) m, & \text { otherwise. }\end{cases}
\end{aligned}
$$

where $0 \leq k<m$. Both $f$ and $g$ are monotone (for example, $f(x, z m+k)=\max (x, \min (z, s(x)))$ ). Consider now the mapping $\langle f, g\rangle: P \times Q \longrightarrow P \times Q$ and recall that $h(x)=f\left(x, \mu_{y} \cdot g(x, y)\right)$. The following holds:

$$
\mu_{y} \cdot g(x, y)=(x+1) m=\left[g_{x}\right]^{m}(\perp), \quad h(x)=f(x,(x+1) m)=s(x), \quad \mu_{x} \cdot h(x)=n=h^{n}(\perp) .
$$

It follows that $\mu \cdot\langle f, g\rangle=\left(n, \mu_{y} \cdot g(n, y)\right)=(n,(n+1) m)$. Finally observe that

$$
\langle f, g\rangle^{(m+1)(n+1)-2}(\perp, \perp)=(n, n m+m-1)<(n,(n+1) m)=\langle f, g\rangle^{(m+1) n+m}(\perp, \perp) .
$$

Proposition 26 (Convergence for (Diag)). Let $f: P \times P \longrightarrow P$ be a monotone function and put $h(x)=_{\operatorname{def}} \mu_{y} . f(x, y)$. Let $n, m \geq 0$ be such that $h(x)=f_{x}^{m}(\perp)$, for each $x \in P$, and $\mu_{x} \cdot h(x)=h^{n}(\perp)$. Then $\mu_{x} . f(x, x)=f^{n m}(\perp, \perp)$. That is, $\operatorname{cl}(f \circ \Delta) \leq \operatorname{cl}(h) \operatorname{cl}_{y}(f)$.

Proof. An easy inspection shows that $\operatorname{cl}(f \circ \Delta)=\operatorname{cl}(\langle f, f\rangle)$ and hence we refer back to Proposition 24. Consider $\mathfrak{f}_{i}, \mathfrak{g}_{i}, \kappa_{i}, \mathfrak{h}_{i}$ as defined in the proof of that Proposition. Here we have $g=f$, so $\mathfrak{g}_{i}=\mathfrak{f}_{i}$ for each $i \geq 0$, and moreover

$$
\mathfrak{h}_{i+1}=f\left(\mathfrak{h}_{i}, \mu_{y} \cdot g\left(\mathfrak{h}_{i}, y\right)\right)=f\left(\mathfrak{h}_{i}, \mu_{y} \cdot f\left(\mathfrak{h}_{i}, y\right)\right)=\mu_{y} \cdot f\left(\mathfrak{h}_{i}, y\right)=\mu_{y} \cdot g\left(\mathfrak{h}_{i}, y\right)=\kappa_{i+1},
$$

so $\mathfrak{h}_{i}=\kappa_{i}$ for each $i \geq 0$. According to the Claim in the proof of Proposition $24, \mathfrak{h}_{i} \leq f_{\psi(i)}$ implies $\kappa_{i+1} \leq \mathfrak{g}_{\psi(i)+m}$; that is, $\mathfrak{h}_{i+1} \leq \mathfrak{f}_{\psi(i)+m}$ since $f=g$. Therefore, letting $\psi(i)=$ def $i m$, we deduce $\mathfrak{h}_{i} \leq \mathfrak{f}_{\psi(i)}$ for all $i \geq 0$ which implies that

$$
\begin{aligned}
\mu_{x} \cdot f(x, x) & =\mu_{x} \cdot \mu_{y} \cdot f(x, y), \\
& =\mu_{x} \cdot h(x), \\
& =\mathfrak{h}_{n}, \\
& \leq \mathfrak{f}_{n m}, \\
\text { since } \mathfrak{h}_{n}=h^{n}(\perp) \text { and we assume that } \mu_{x} \cdot h(x)=h^{n}(\perp), & \text { since } h(x)=\mu_{y} \cdot f(x, y),
\end{aligned}
$$

as needed. 


\subsection{Results for Heyting algebras}

In many cases, formula (23) given in Proposition 24 does not yield a tight upper bound. In particular this happens when we want to estimate the convergence of weakly negative formulas whose least fixed-points can be computed by using the Bekic property, as we have seen in the previous Section 6.4.

In order to improve the upper bound given in (23), we need the following observation.

Lemma 27. Let $\langle f, g\rangle: P \times Q \longrightarrow P \times Q$ be a monotone mapping, put $h(x)=\operatorname{def} f\left(x, \mu_{y} . g(x, y)\right)$, let $m, n \geq 0$ be such that $\mu_{y} . g(x, y)=g_{x}^{m}(\perp)$ for each $x \in P$ and $\mu_{x} . h(x)=h^{n}(\perp)$. Under these hypothesis we have

$$
\pi_{1}(\mu .\langle f, g\rangle)=\pi_{1}\left(\langle f, g\rangle^{n(m+1)}(\perp, \perp)\right) .
$$

Proof. Using the same notation as in the proof of Proposition 24, it is enough to observe that

$$
\mu .\langle f, g\rangle \leq\left\langle\mathfrak{f}_{\psi(n)}, \mathfrak{g}_{\psi(n)+m}\right\rangle \leq \mu .\langle f, g\rangle,
$$

with $\psi(n)=n(m+1)$, so $\pi_{1}\left(\langle f, g\rangle^{\psi(n)}\right)=\mathfrak{f}_{\psi(n)}=\pi_{1}(\mu .\langle f, g\rangle)$.

By using the lemma, we are going to obtain the tight upper bound for the least solution of system of equations used for weakly negative formula-terms.

Proposition 28. Consider a monotone $\left\langle f_{1}, \ldots, f_{k}\right\rangle: Q \times P^{k} \longrightarrow P^{k}$ and suppose that, for each $g: Q \times P^{k} \longrightarrow P$ in the cone generated by the functions $\left\{f_{1}, \ldots, f_{k}\right\} \cup\{\perp\}, \mathrm{cl}_{x_{i}}(g) \leq 1$ for each $i=1, \ldots, k$. Then $\mu .\left\langle f_{1}, \ldots, f_{k}\right\rangle_{q} \leq$ $\left\langle f_{1}, \ldots, f_{k}\right\rangle_{q}^{k}(\perp)$ for each $q \in Q$ or, said otherwise, $\mathrm{cl}_{\left(x_{1}, \ldots, x_{k}\right)}\left(\left\langle f_{1}, \ldots, f_{k}\right\rangle\right) \leq k$.

Proof. The proof is by induction on $k \geq 1$. When $k=1$ then, $\mathrm{cl}_{x_{1}}\left(f_{1}\right) \leq 1$ by assumption.

Now suppose that $k>1$ and that the property holds for all monotone functions $\left\langle f_{i_{1}}, \ldots, f_{i_{l}}\right\rangle: Q \times P^{\ell} \longrightarrow P^{\ell}$ with $\ell<k$.

By the induction hypothesis, $\mathrm{cl}_{\left(x_{2}, \ldots, x_{k}\right)}\left(\left\langle f_{2}, \ldots, f_{k}\right\rangle\right) \leq k-1$. For each $q \in Q$ consider the function $h_{q}$ defined by $h_{q}\left(x_{1}\right)=$ def $_{1}\left(q, x_{1},\left\langle f_{2}, \ldots, f_{k}\right\rangle_{\left(q, x_{1}\right)}^{k}(\perp)\right) ; h_{q}$ belongs to the cone generated by $\left\{f_{1}, \ldots, f_{k}\right\} \cup\{\perp\}$ and therefore $\mathrm{cl}_{x_{1}}\left(h_{q}\right) \leq 1$ by assumption. We can therefore apply Lemma 27 (with $f=h_{q}, g=\left\langle f_{2}, \ldots, f_{k}\right\rangle, n=1$ and $m=k-1$ ) to deduce that, for each $q \in Q$,

$$
\pi_{1}\left(\mu .\left\langle f_{1}, \ldots, f_{k}\right\rangle_{q}\right) \leq \pi_{1}\left(\left\langle f_{1}, \ldots, f_{k}\right\rangle_{q}^{1 \cdot(k-1+1)}\right)(\perp)=\pi_{1}\left(\left\langle f_{1}, \ldots, f_{k}\right\rangle_{q}^{k}(\perp)\right) .
$$

In a similar way we deduce

$$
\pi_{i}\left(\mu .\left\langle f_{1}, \ldots, f_{k}\right\rangle_{q}\right) \leq \pi_{i}\left(\left\langle f_{1}, \ldots, f_{k}\right\rangle_{q}^{k}(\perp)\right),
$$

for each $i=1, \ldots, k$, and therefore $\mu .\left\langle f_{1}, \ldots, f_{k}\right\rangle_{q} \leq\left\langle f_{1}, \ldots, f_{k}\right\rangle_{q}^{k}(\perp)$.

To see that the bound given in the previous proposition is tight it is enough to compute the least solution of the system of equations

$$
\left\{x_{i}=\left\{a_{i}\right\} \cup x_{i-1 \bmod k} \mid i=0, \ldots, k-1\right\},
$$

in the powerset of $P\left(\left\{a_{1}, \ldots, a_{k}\right\}\right)$.

We can finally give a better upper bound to closure ordinals of weakly negative formula-terms. Manuscript submitted to ACM 
Proposition 29. Let $\phi(x)$ be a weakly negative formula-term, so that we have a decomposition of the form (20). Then $\phi(x)$ converges to its least fixed-point in at most $n+1$ steps.

Proof. By combining Propositions 10 and 28, we have

$$
v \cdot\left(\left\langle\psi_{i} \mid i=1, \ldots, n\right\rangle \circ \psi_{0}\right)=\left(\left\langle\psi_{i} \mid i=1, \ldots, n\right\rangle \circ \psi_{0}\right)^{n}(T) .
$$

Considering that

$$
\mu . \phi=\mu .\left(\psi_{0} \circ\left\langle\psi_{i} \mid i=1, \ldots, n\right\rangle\right)=\psi_{0}\left(\nu \cdot\left(\left\langle\psi_{i} \mid i=1, \ldots, n\right\rangle \circ \psi_{0}\right)\right)
$$

we can use equation (24) and Proposition 22 to deduce that

$$
\mu . \phi=\left(\psi_{0} \circ\left\langle\psi_{i} \mid i=1, \ldots, n\right\rangle\right)^{n+1}(\perp) .
$$

We can expect that other formulas for fixed-points have a counterpart with closure ordinals. This is the case for equation (9). To give an account of it, we firstly prove a Lemma.

Lemma 30. Let $H$ be a Heyting algebra and let $f$ and $g$ be monotone polynomials on $H$. For every pair of natural numbers $n, m$ such that $n+m \geq 1, f^{n}(\perp) \wedge g^{m}(\perp) \leq(f \wedge g)^{n+m-1}(\perp)$.

Proof. Let $h$ denote the polynomial $f \wedge g$ on $H$. We prove the result by induction on $k=n+m \geq 1$.

If $n+m=1$, then either $n=0$ or $m=0$. In this case either $f^{n}(\perp)=\perp$ or $g^{m}(\perp)=\perp$, so $f^{n}(\perp) \wedge g^{m}(\perp)=\perp$, so the result is obvious.

Now suppose that the result holds for any pair of numbers $n^{\prime}, m^{\prime}$ such that $1 \leq n^{\prime}+m^{\prime} \leq k$. Let $m$ and $n$ be such that $m+n=k+1$. The following holds:

$$
\begin{aligned}
f^{n}(\perp) \wedge g^{m}(\perp)= & f^{n}(\perp) \wedge g^{m}(\perp) \wedge f^{n}(\perp) \wedge g^{m}(\perp) \\
& \leq f\left(f^{n-1}(\perp) \wedge g^{m}(\perp)\right) \wedge g\left(f^{n}(\perp) \wedge g^{m-1}(\perp)\right), \\
& \leq f\left(h^{k-1}(\perp)\right) \wedge g\left(h^{k-1}(\perp)\right), \\
& =h^{k}(\perp)=h^{n+m-1}(\perp) .
\end{aligned}
$$

by the IH,

Next we show that $\operatorname{cl}(f \wedge g)<\operatorname{cl}(f)+\operatorname{cl}(g)$. This relation holds when $\operatorname{cl}(f)+\operatorname{cl}(g)>0$; in order to settle trivial cases, we let $h^{k}(\perp)=\perp$ for $k<0$ in the statement of the Proposition below.

Proposition 31. Let $H$ be an Heyting algebra. If $f$ and $g$ are monotone polynomials on $H$ such that $\mu_{x} \cdot f(x)=f^{m}(\perp)$ and $\mu_{x} \cdot g(x)=g^{n}(\perp)$, then $\mu_{x} \cdot(f \wedge g)(x)=(f \wedge g)^{m+n-1}(\perp)$. That is, $\operatorname{cl}(f \wedge g) \leq \mathrm{cl}(f)+\operatorname{cl}(g)-1$.

Proof. Let $h(x)=f(x) \wedge g(x)$ and compute as follows:

$$
\begin{aligned}
h^{n+m-1}(\perp) & \leq \mu_{x} \cdot h(x)=\mu_{x} \cdot(f(x) \wedge g(x)) \\
& =\mu_{x} \cdot f(x) \wedge \mu_{x} \cdot g(x) \\
& =f^{n}(\perp) \wedge g^{m}(\perp) \\
& \leq h^{n+m-1}(\perp)
\end{aligned}
$$

so we have the equality $\mu_{x} \cdot h(x)=h^{n+m-1}(\perp)$.

Proposition 32. The upper bound $m+n-1$ given in Proposition 31 is tight. 
Proof. Observe that if $H$ is a Heyting algebra which is a chain, then $x \rightarrow a=\mathrm{\top}$, if $x \leq a$, and $x \rightarrow a=a$, otherwise. If $H$ is such an Heyting algebra which contains the chain $\perp \leq a_{0}<a_{1}<a_{2}<\ldots<a_{k-1}<a_{k}=\mathrm{T}$, let

$$
f_{j}(x)=_{\text {def }}\left(x \rightarrow a_{j-1}\right) \rightarrow a_{j}, \quad \text { for } j=1, \ldots, k-1 .
$$

We have then, for each $i, j$ with $0 \leq i \leq k$ and $1 \leq j<k$,

$$
f_{j}\left(a_{i}\right)=\left(a_{i} \rightarrow a_{j-1}\right) \rightarrow a_{j}= \begin{cases}a_{j} & i<j, \\ \top & i \geq j .\end{cases}
$$

Define then

$$
f_{a_{0}, a_{1}, \ldots, a_{k-1}}(x)=_{\text {def }} \bigwedge_{j=1, \ldots, k-1} f_{j}(x) .
$$

Claim. For each $i=1, \ldots, k$ we have

$$
f_{a_{0}, a_{1}, \ldots a_{k-1}}^{i}(\perp)=a_{i}
$$

Proof of Claim. The relation trivially holds for $i=1$. Assuming it holds for $i$, we have

$$
f_{a_{0}, a_{1}, \ldots, a_{k-1}}^{i+1}(\perp)=\bigwedge_{j=1, \ldots, k-1} f_{j}\left(a_{i}\right)=\bigwedge_{i<j \leq k-1} f_{j}\left(a_{i}\right)=\bigwedge_{i<j \leq k-1} a_{j}=a_{i+1} .
$$

Observe that the above relation holds also when $i+1=k$, in which case $\{j \mid i<j \leq k-1\}=\emptyset$, so the meet above is empty, so equal to $\mathrm{T}=a_{k}$.

It follows from the Claim that $\mu_{x} \cdot f_{a_{0}, a_{1}, \ldots, a_{k-1}}(x)=f_{a_{0}, a_{1}, \ldots, a_{k-1}}^{k}(\perp)=\top>a_{k-1}=f^{k-1}(\perp)$.

Now assume that $H$ contains the chain $\perp \leq a_{0}<a_{1}<a_{2}<\ldots<a_{m+n-2}<a_{m+n-1}=\mathrm{T}$. We have then

$$
f_{a_{0}, a_{1}, \ldots, a_{n+m-2}}(x)=f_{a_{0}, a_{1}, \ldots, a_{n-1}}(x) \wedge f_{a_{n-1}, a_{n}, \ldots, a_{n+m-2}}(x),
$$

with

$$
\begin{aligned}
\mu_{x} \cdot f_{a_{0}, a_{1}, \ldots, a_{n-1}}(x) & =f_{a_{0}, a_{1}, \ldots, a_{n-1}}^{n}(\perp), \\
\mu_{x} \cdot f_{a_{n-1}, a_{n}, \ldots, a_{n+m-2}}(x) & =f_{a_{n-1}, a_{n}, \ldots, a_{n+m-2}}^{m}(\perp), \\
\mu_{x} \cdot f_{a_{0}, a_{1}, \ldots, a_{n+m-2}}(x) & =f_{a_{0}, a_{1}, \ldots, a_{n+m-2}}^{n+m-1}(\perp)>f_{a_{0}, a_{1}, \ldots, a_{n+m-2}}^{n+m-2}(\perp) .
\end{aligned}
$$

Finally, we provide a tight upper bound for closure ordinals of disjunctive formulas.

Proposition 33. If $\phi$ is a disjunctive formula, then

$$
\mu_{x} \cdot \phi(x)=\phi^{n+1}(\perp),
$$

where $n$ is the cardinality of the set $\operatorname{Head}(\phi)$.

Proof. By Proposition 17 we know that $\mu_{x} . \phi(x)=\left[\bigwedge_{i=1, \ldots, n} \alpha_{i}\right]\left(\bigvee_{\beta \in \operatorname{Side}(\phi)} \beta\right)$. We have seen that, for $\alpha \in$ $\operatorname{Head}(\phi),[\alpha] x \leq \phi(x)$ and, similarly, $\beta \vee x \leq \phi(x)$ for $\beta \in \operatorname{Side}(\phi)$. Thus we have

$$
\bigvee_{\beta \in \operatorname{Side}(\phi)} \beta=\bigvee_{\beta \in \operatorname{Side}(\phi)} \beta \vee \perp \leq \phi(\perp) .
$$

Manuscript submitted to ACM 
Let $\operatorname{Head}(\phi)=\left\{\alpha_{1}, \ldots, \alpha_{n}\right\}$ and suppose that

$$
\left[\alpha_{i}\right] \ldots\left[\alpha_{1}\right]\left(\bigvee_{\beta \in \operatorname{Side}(\phi)} \beta\right) \leq \phi^{i+1}(\perp) .
$$

Then

$$
\left[\alpha_{i+1}\right]\left[\alpha_{i}\right] \ldots\left[\alpha_{1}\right]\left(\bigvee_{\beta \in \operatorname{Side}(\phi)} \beta\right) \leq\left[\alpha_{i+1}\right]\left(\phi^{i+1}(\perp)\right) \leq \phi\left(\phi^{i+1}(\perp)\right)=\phi^{i+2}(\perp)
$$

Whence

$$
\mu_{x} . \phi(x)=\left[\bigwedge_{i=1, \ldots, n} \alpha_{i}\right]\left(\bigvee_{\beta \in \operatorname{Side}(\phi)} \beta\right)=\left[\alpha_{n}\right] \ldots\left[\alpha_{1}\right]\left(\bigvee_{\beta \in \operatorname{Side}(\phi)} \beta\right) \leq \phi^{n+1}(\perp)
$$

We prove the next proposition using Kripke models, see e.g. [Bezhanishvili and de Jongh 2006, §3.3]. A Kripke model is a triple $\langle W, \leq, V\rangle$ where $\leq$ is an ordering on the set $W$ and $V$ is a downward closed valuation of atomic formulas (that is, $w \in V(p)$ and $w^{\prime} \leq w$ implies $w \in V(p)$, for each atomic formula $p$ ). The forcing relation $\Vdash$ between worlds and formulas is defined as usual for atomic formulas and formulas of the form $\perp, \phi \vee \psi, \top, \phi \wedge \psi$. For implication formulas we have

$$
w \Vdash \phi \rightarrow \psi \quad \text { iff } \quad \forall w^{\prime} \leq w, w^{\prime} \Vdash \phi \Rightarrow w^{\prime} \Vdash \psi .
$$

Proposition 34. The above upper bound given in equation (25) is tight.

Proof. For each $n \geq 0$, consider the formula

$$
\phi_{n}(x)=\operatorname{def} b \vee \bigvee_{i=1, \ldots, n} a_{i} \rightarrow x,
$$

and the Kripke model $K_{n}=\langle P(\{1, \ldots, n\}), \subseteq, V\rangle$ with $V(b)=\{\emptyset\}$ and, for $i=1, \ldots, n, V\left(a_{i}\right)=\{s \subseteq\{1, \ldots, n\} \mid i \notin s\}$. We have therefore $s \Vdash a_{i}$ iff $i \notin s$, for $s \in P(\{1, \ldots, n\})$. Let us compute the value of $\phi_{n}(x)$.

$$
s \Vdash a_{i} \rightarrow x \text { iff } \forall s^{\prime} \subseteq s, i \notin s^{\prime} \Rightarrow s^{\prime} \Vdash x \text { iff } s \backslash\{i\} \Vdash x,
$$

whence

$$
s \Vdash \phi_{n}(x) \text { iff either } s=\emptyset \text { or } s \backslash\{i\} \Vdash x \text {, for some } i \in\{1, \ldots, n\} \text {. }
$$

Thus it is immediate to see that

$$
\phi_{n}^{k+1}(\emptyset)=\{s \subseteq\{1, \ldots, n\} \mid \operatorname{card} s \leq k\}
$$

so that $\phi_{n}$ converges in no less than $n+1$ steps.

\section{RUITENBURG'S NUMBERS FOR STRONGLY POSITIVE FORMULAS}

Let $\phi$ be a formula of the Intuitionistic Propositional Calculus (possibly) containing the variable $x$. By $\phi^{n}$ we denote the iterated substitution of $x$ in $\phi$ for $\phi$, defined by induction by $\phi^{0}={ }_{\operatorname{def}} x$ and $\phi^{n+1}={ }_{\operatorname{def}} \phi\left[\phi^{n} / x\right]$. We let $\rho(\phi)$ be the least non-negative integer $n$ such that the relation $\phi^{n+2}=\phi^{n}$ holds; $\rho(\phi)$ is defined for any formula $\phi$ of the Intuitionistic Propositional Calculus, by [Ruitenburg 1984], and moreover $\operatorname{cl}(\phi) \leq \rho(\phi)$. A fine analysis of Ruitenburg's work shows that $\rho(\phi) \leq 2 n+2$, where $n$ counts the implication subformulas and the propositional variables in $\phi$. 
The tools developed until now allow to construct an upper bound for $\mathrm{cl}(\phi)$ for any formula $\phi$ of the Intuitionistic Propositional Calculus, yet the bound so obtained is exponential in the size of $\phi$; thus, in view of the relation $\operatorname{cl}(\phi) \leq$ $\rho(\phi) \leq 2 n+2$, it is not optimal. We exemplify this point. Let $\phi$ be a strongly positive formula and let $n$ be its size (the number of all symbols and propositional variables in $\phi$ ). When transforming $\phi$ into a conjunction of disjunctive formulas, so

$$
\phi=\underset{\mathrm{IPC}}{ } \bigwedge_{i=1, \ldots ., k} \phi_{i}
$$

the number $k$ of conjuncts might be exponentially bigger than $n$. Say that $\operatorname{cl}\left(\phi_{i}\right) \leq N$ for each $i=1, \ldots, k$. An iterated application of Proposition 31 yields the following upper bound:

$$
\operatorname{cl}(\phi)=\operatorname{cl}\left(\bigwedge_{i} \phi_{i}\right) \leq 1+\sum_{i=1, \ldots, k}\left(\operatorname{cl}\left(\phi_{i}\right)-1\right) \leq 1+k(N-1),
$$

which depends on some possibly very large $k$.

From now on, our goal shall be to give an upper bound for $\operatorname{cl}(\phi)$ when $\phi$ is a formula such as the one in (either side of) equation (26). Since our proofs actually yield upper bounds for Ruitenburg's numbers $\rho(\phi)$ (and a proof of Ruitenburg's theorem for these formulas) we present our results directly as bounds for the numbers $\rho(\phi)$.

While the procedure that transforms a strongly positive formula $\phi$ (say as the one on the left of (26)) into a conjunction of disjunctive formulas $\phi_{i}$ (as the one on the right of (26)) might exponentially increase the size of the formula, as argued above, it does not increase the number of head subformulas nor the number of side subformulas. Therefore we give bounds as functions of these two parameters, which eventually ensures an upper bound to Ruitenburg's numbers of strongly positive formulas which is quadratic in the size of the formulas. In view of obtaining these upper bounds we can (and shall) suppose that all the head or side subformulas are propositional variables.

In the following we let $\mathcal{A}={ }_{\text {def }}\left\{\alpha_{1}, \ldots, \alpha_{N}\right\}$ and $\mathcal{B}=$ def $_{\text {d }}\left\{\beta_{1}, \ldots, \beta_{M}\right\}$ be two (finite) disjoint sets of propositional variables; we also suppose that the special propositional variable $x$ does not belong to either of $\mathcal{A}$ and $\mathcal{B}$. We consider formulas of the Intuitionistic Propositional Calculus generated by the following grammar:

$$
\phi \Rightarrow x|[A] \phi|(\bigvee B) \vee \phi \mid \phi \vee \phi
$$

where $A \subseteq \mathcal{A}, B \subseteq \mathcal{B}$ and, as before, $[A] \phi=\wedge A \rightarrow \phi$. That is, formulas generated by the above grammar are disjunctive formulas, as defined by the grammar (15), whose head formulas are conjunctions of propositional variables from $\mathcal{A}$, and whose side formulas are disjunctions of propositional variables from $\mathcal{B}$. We let $\operatorname{Disj}(\mathcal{A}, \mathcal{B})$ be the set of formulas generated by (27). We consider formulas in $\operatorname{Disj}(\mathcal{A}, \mathcal{B})$ as elements of $\mathrm{F}_{\mathrm{H}}\left[\alpha_{1}, \ldots, \alpha_{N}, \beta_{1}, \ldots, \beta_{M}, x\right]$, the free Heyting algebra on the generators $\alpha_{1}, \ldots, \alpha_{N}, \beta_{1}, \ldots, \beta_{M}, x$. Substitution of a formula $\psi$ for the variable $x$ in a formula $\phi$, usually noted by $\phi[\psi / x]$, yields a monoid structure on $\mathrm{F}_{\mathrm{H}}\left[\alpha_{1}, \ldots, \alpha_{N}, \beta_{1}, \ldots, \beta_{M}, x\right]$. We write $\phi \circ \psi$ for $\phi[\psi / x]$ or sometimes, $\phi(\psi)$. Since formulas in $\operatorname{Disj}(\mathcal{A}, \mathcal{B})$ are closed under substitution, $\operatorname{Disj}(\mathcal{A}, \mathcal{B})$ is a submonoid of $\mathrm{F}_{\mathrm{H}}\left[\alpha_{1}, \ldots, \alpha_{N}, \beta_{1}, \ldots, \beta_{M}, x\right] . \operatorname{Disj}(\mathcal{A}, \mathcal{B})$ is actually an ordered submonoid, meaning that the following clause is valid:

$$
\phi \leq \phi^{\prime} \text { and } \psi \leq \psi^{\prime} \text { implies } \phi \circ \psi \leq \phi^{\prime} \circ \psi^{\prime} .
$$

Manuscript submitted to ACM 
This is mainly because the variable $x$ never occurs under the left side of any implication in a formula in $\operatorname{Disj}(\mathcal{A}, \mathcal{B})$. Moreover, formulas are inflating, meaning that

$$
x \leq \phi, \quad \text { for each } \phi \in \operatorname{Disj}(\mathcal{A}, \mathcal{B}) .
$$

\subsection{The support of a formula}

We define next two functions, $\operatorname{Supp}_{\mathcal{A}}$ and $\operatorname{Supp}_{\mathcal{B}}$, with domain $\operatorname{Disj}(\mathcal{A}, \mathcal{B})$ and codomain $P(\mathcal{A})$ and $P(\mathcal{B})$, respectively:

$$
\begin{aligned}
& \operatorname{Supp}_{\mathcal{A}}(x)=_{\text {def }} \emptyset, \\
& \operatorname{Supp}_{\mathcal{B}}(x)={ }_{\text {def }} \emptyset, \\
& \operatorname{Supp}_{\mathcal{A}}([A] \phi)=\operatorname{def}_{\operatorname{def}} A \operatorname{Supp}_{\mathcal{A}}(\phi) \text {, } \\
& \operatorname{Supp}_{\mathcal{B}}([A] \phi)=_{\text {def }} \operatorname{Supp}_{\mathcal{B}}(\phi), \\
& \operatorname{Supp}_{\mathcal{A}}((\bigvee B) \vee \phi)=_{\operatorname{def}} \operatorname{Supp}_{\mathcal{A}}(\phi), \\
& \operatorname{Supp}_{\mathcal{B}}((\bigvee B) \vee \phi)=_{\operatorname{def}} B \cup \operatorname{Supp}_{\mathcal{B}}(\phi), \\
& \operatorname{Supp}_{\mathcal{A}}\left(\phi_{0} \vee \phi_{1}\right)==_{\text {def }} \operatorname{Supp}_{\mathcal{A}}\left(\phi_{0}\right) \cup \operatorname{Supp}_{\mathcal{A}}\left(\phi_{1}\right),
\end{aligned}
$$

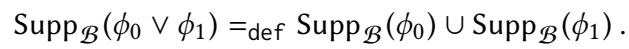

We also let

$$
\operatorname{Supp}(\phi)=_{\operatorname{def}}\left(\operatorname{Supp}_{\mathcal{A}}(\phi), \operatorname{Supp}_{\mathcal{B}}(\phi)\right)
$$

so $\operatorname{Supp}(\phi) \in P(\mathcal{A}) \times P(\mathcal{B})$.

\subsection{Word formulas}

In the inverse direction, given $(A, B) \in P(\mathcal{A}) \times P(\mathcal{B})$, we define

$$
\phi_{(A, B)}=_{\operatorname{def}}[A](\bigvee B \vee x) .
$$

Notice that $\phi_{(\emptyset, B)}=[\top](\vee B \vee x)=\vee B \vee x$ and $\phi_{(A, \emptyset)}=[A](\perp \vee x)=[A] x$. Let us develop the basic properties of the formulas $\phi_{(A, B)}$.

Proposition 35. For each $\left(A_{0}, B_{0}\right),\left(A_{1}, B_{1}\right) \in P(\mathcal{A}) \times P(\mathcal{B})$ and each $\phi \in \operatorname{Disj}(\mathcal{A}, \mathcal{B})$,

$$
\begin{aligned}
\phi_{\left(A_{0}, \emptyset\right)} \circ \phi_{\left(A_{1}, B_{1}\right)} & =\phi_{\left(A_{0} \cup A_{1}, B_{1}\right)}, \\
\phi_{\left(A_{0}, B_{0}\right)} \circ \phi_{\left(\emptyset, B_{1}\right)} & =\phi_{\left(A_{0}, B_{0} \cup B_{1}\right)}, \\
\phi_{\left(A_{0}, B_{0}\right)} \circ \phi \circ \phi_{\left(A_{1}, B_{1}\right)} & =\phi_{\left(A_{0}, B_{0} \backslash B_{1}\right)} \circ \phi \circ \phi_{\left(A_{1} \backslash A_{0}, B_{1}\right)} .
\end{aligned}
$$

Proof. The first two properties are immediate from the definition of $\phi_{(A, B)}$. For the third, notice that

$$
\begin{aligned}
\phi_{\left(A_{0}, B_{0}\right)} \circ \phi & \circ \phi_{\left(A_{1}, B_{1}\right)} \\
& =\left[A_{0}\right]\left(\bigvee B_{0} \vee \phi\left(\left[A_{1}\right]\left(\bigvee B_{1} \vee x\right) / x\right)\right) \\
& =\left[A_{0} \cap A_{1}\right]\left[A_{0} \backslash A_{1}\right]\left(\bigvee B_{0} \vee \phi\left(\left[A_{0} \cap A_{1}\right]\left[A_{1} \backslash A_{0}\right]\left(\bigvee B_{1} \vee x\right) / x\right)\right) \\
& =\left[A_{0} \cap A_{1}\right]\left[A_{0} \backslash A_{1}\right]\left(\bigvee B_{0} \vee \phi\left(\left[A_{1} \backslash A_{0}\right]\left(\bigvee B_{1} \vee x\right) / x\right)\right), \quad \text { by Lemma } 7, \\
& =\left[A_{0}\right]\left(\bigvee B_{0} \vee \phi\left(\left[A_{1} \backslash A_{0}\right]\left(\bigvee B_{1} \vee x\right) / x\right)\right) \\
& =\left[A_{0}\right]\left(\bigvee B_{0} \backslash B_{1} \vee \phi\left(\left[A_{1} \backslash A_{0}\right]\left(\bigvee B_{1} \vee x\right)\right)\right), \\
& =\phi\left(A_{0}, B_{0} \backslash B_{1}\right) \circ \phi \circ \phi\left(A_{1} \backslash A_{0}, B_{1}\right) .
\end{aligned}
$$

An immediate consequence of the proposition is the following: 
LemmA 36. For each $(A, B) \in P(\mathcal{A}) \times P(\mathcal{B}), \phi_{(A, B)}^{2}=\phi_{(A, B)}$, so $\rho\left(\phi_{(A, B)}\right)=1$.

We extend the definition of the correspondence sending $(A, B) \in P(\mathcal{A}) \times P(\mathcal{B})$ to $\phi_{(A, B)} \in \operatorname{Disj}(\mathcal{A}, \mathcal{B})$ to the set of all words over the alphabet $P(\mathcal{A}) \times P(\mathcal{B})$-that shall be noted by $(P(\mathcal{A}) \times P(\mathcal{B}))^{*}$, as usual. Syntactically, this amounts to defining $\phi_{w}$ for each $w \in(\mathcal{A} \times \mathcal{B})^{*}$, as follows:

$$
\phi_{\epsilon}=_{\text {def }} x, \quad \phi_{(A, B) w}={ }_{\text {def }} \phi_{(A, B)} \circ \phi_{w} .
$$

We call a formula of the form $\phi_{w}$ for some $w \in(P(\mathcal{A}) \times P(\mathcal{B}))^{*}$ a word formula.

Lemma 37. For each $w \in(P(\mathcal{A}) \times P(\mathcal{B}))^{*}, \phi_{w} \in \operatorname{Disj}(\mathcal{A}, \mathcal{B})$. Moreover, if $w=\left(A_{1}, B_{1}\right) \ldots\left(A_{k}, B_{k}\right)$, then

$$
\begin{aligned}
\operatorname{Supp}\left(\phi_{w}\right) & =\left(\bigcup_{i=1 \ldots, k} A_{i}, \bigcup_{i=1 \ldots, k} B_{i}\right), \\
\phi_{w} \leq \phi_{w}^{2} & =\phi_{\operatorname{Supp}\left(\phi_{w}\right)}, \\
\rho\left(\phi_{w}\right) & \leq 2 .
\end{aligned}
$$

Proof. The first statement is a consequence of formulas of $\operatorname{Disj}(\mathcal{A}, \mathcal{B})$ being closed under substitution. Equation (30) is easily proved by induction. The relation $\phi_{w} \leq \phi_{w}^{2}$ is an easy consequence of conditions (28) and (29). $\phi_{w}^{2}=\phi_{\operatorname{Supp}}\left(\phi_{w}\right)$ is obtained by iteratively applying the relations in Proposition 35. Finally we argue that $\phi_{w}^{3}=\phi_{w}^{2}$ (so $\left.\rho\left(\phi_{w}\right)=2\right)$ as follows:

$$
\phi_{w}^{2} \leq \phi_{w}^{3} \leq \phi_{w}^{4}=\phi_{\operatorname{Supp}\left(\phi_{w}\right)}^{2}=\phi_{\operatorname{Supp}\left(\phi_{w}\right)}=\phi_{w}^{2}
$$

In view of (30), let us define

$$
\operatorname{Supp}\left(\left(A_{1}, B_{1}\right) \ldots\left(A_{k}, B_{k}\right)\right)=\operatorname{def}\left(\bigcup_{i=1 \ldots, k} A_{i}, \bigcup_{i=1 \ldots, k}^{\bigcup} B_{i}\right),
$$

so $\operatorname{Supp}(w)=\operatorname{Supp}\left(\phi_{w}\right)$

Lemma 38. For each $\phi \in \operatorname{Disj}(\mathcal{A}, \mathcal{B}), \phi \leq \phi_{\operatorname{Supp}(\phi)}$.

Proof. We inductively define, for each $\phi \in \operatorname{Disj}(\mathcal{A}, \mathcal{B})$, a word $w(\phi)$ such that $\phi \leq \phi_{w(\phi)}$ and $\operatorname{Supp}(\phi)=\operatorname{Supp}(w(\phi))$. Then, using equation (31), we deduce

$$
\phi \leq \phi_{w(\phi)} \leq \phi_{\operatorname{Supp}(w(\phi))}=\phi_{\operatorname{Supp}(\phi)}
$$

We let $w(x)=_{\text {def }}(\emptyset, \emptyset), w([A] \phi)=_{\text {def }}(A, \emptyset) w(\phi), w(\vee B \vee \phi)=_{\text {def }}(\emptyset, B) w(\phi)$, and

$$
w\left(\phi_{0} \vee \phi_{1}\right)=_{\operatorname{def}} w\left(\phi_{0}\right) w\left(\phi_{1}\right) .
$$

By induction, it is proved that $\phi \leq \phi_{w(\phi)}$ and $\operatorname{Supp}(\phi)=\operatorname{Supp}(w(\phi))$, the only non-obvious inductive case being the last, which we prove next. For $i=0,1$, let $w_{i}=$ def $w\left(\phi_{i}\right)$ and suppose that $\phi_{i} \leq \phi_{w_{i}}$ and $\operatorname{Supp}\left(\phi_{i}\right)=\operatorname{Supp}\left(\phi_{w_{i}}\right)$. Then $\operatorname{Supp}(\phi)=\operatorname{Supp}\left(\phi_{0}\right) \cup \operatorname{Supp}\left(\phi_{1}\right)=\operatorname{Supp}\left(\phi_{w_{0}}\right) \cup \operatorname{Supp}\left(\phi_{w_{1}}\right)=\operatorname{Supp}\left(\phi_{w_{0}} \circ \phi_{w_{1}}\right)=\operatorname{Supp}\left(\phi_{w_{0} w_{1}}\right)$ and

$$
\phi=\phi_{0} \vee \phi_{1} \leq \phi_{0} \circ \phi_{1} \leq \phi_{w_{0}} \circ \phi_{w_{1}}=\phi_{w(\phi)},
$$

where the relation $\phi_{0} \vee \phi_{1} \leq \phi_{0} \circ \phi_{1}$ is a consequence of $\phi_{i}, i=0,1$, being inflating.

Manuscript submitted to ACM 
We shall see later-as a particular instance of Theorem 45-that $\phi^{n}=\phi_{\operatorname{Supp}(\phi)}$ for some $n$, and for each $\phi \in \operatorname{Disj}(\mathcal{A}, \mathcal{B})$. That is, $\phi_{\mathrm{Supp}(\phi)}$ yields a closed expression of the formula $\phi^{\rho(\phi)}$. We shall further exploit word formulas in the rest of the section and heavily rely on the next observation.

Definition 5. For $(A, B) \in P(\mathcal{A}) \times P(\mathcal{B})$ and $w=\left(A_{1}, B_{1}\right), \ldots,\left(A_{k}, B_{k}\right) \in(P(\mathcal{A}) \times P(\mathcal{B}))^{*}$, we let

$$
(A, B) \triangleleft\left(A_{1}, B_{1}\right) \ldots\left(A_{k}, B_{k}\right) \text { iff } \exists l \leq k \text { s.t. } A \subseteq \bigcup_{j \leq l} A_{j} \text { and } B \subseteq \bigcup_{j \geq l} B_{j} .
$$

Proposition 39. If $(A, B) \triangleleft w$, then $\phi_{(A, B)} \leq \phi_{w}$.

Proof. Let $w=\left(A_{1}, B_{1}\right), \ldots,\left(A_{k}, B_{k}\right)$ and let $\ell$ be such that $(33)$ holds. Define $w_{L}=\left(A_{1}, B_{1}\right), \ldots,\left(A_{\ell}, \emptyset\right)$ and $w_{R}=\left(\emptyset, B_{\ell}\right), \ldots,\left(A_{n}, B_{k}\right)$. Observe that

$$
\phi_{(A, \emptyset)} \leq \phi_{\left(\bigcup_{i=1 \ldots \ell} A_{i}, \emptyset\right)}=\phi_{\left(A_{1}, \emptyset\right)} \circ \ldots \circ \phi_{\left(A_{\ell}, \emptyset\right)} \leq \phi_{\left(A_{1}, B_{1}\right)} \circ \ldots \circ \phi_{\left(A_{\ell-1}, B_{\ell-1}\right)} \circ \phi_{\left(A_{\ell}, \emptyset\right)}=\phi_{w_{L}},
$$

and, similarly, $\phi_{(\emptyset, B)} \leq \phi_{w_{R}}$. It follows that $\phi_{(A, B)}=\phi_{(A, \emptyset)} \circ \phi_{(\emptyset, B)} \leq \phi_{w_{L}} \circ \phi_{w_{R}}=\phi_{w}$.

\subsection{Conjunctions of star formulas}

In the next definition, if $X, Y \subseteq(P(\mathcal{A}) \times P(\mathcal{B}))^{*}$, then we let

$$
X \cdot Y=_{\operatorname{def}}\{w v \mid w \in X, v \in Y\} .
$$

Definition 6. The set $\operatorname{Branches}(\phi) \subseteq(P(\mathcal{A}) \times P(\mathcal{B}))^{*}$ of branches of $\phi \in \operatorname{Disj}(\mathcal{A}, \mathcal{B})$ is defined by induction:

$$
\begin{aligned}
\operatorname{Branches}(x) & =\operatorname{def}_{\operatorname{def}}\{\epsilon\} \\
\operatorname{Branches}(\bigvee B \vee \phi) & ={ }_{\operatorname{def}}\{(\emptyset, B)\} \cdot \operatorname{Branches}(\phi) \\
\operatorname{Branches}([A] \phi) & ={ }_{\operatorname{def}}\{(A, \emptyset)\} \cdot \operatorname{Branches}(\phi) \\
\operatorname{Branches}\left(\phi_{0} \vee \phi_{1}\right) & ={ }_{\text {def }} \operatorname{Branches}\left(\phi_{0}\right) \cup \operatorname{Branches}\left(\phi_{1}\right) .
\end{aligned}
$$

The formula $\operatorname{br}(\phi)$ is then defined as follows:

$$
\operatorname{br}(\phi)=_{\text {def }} \bigvee\left\{\phi_{w} \mid w \in \operatorname{Branches}(\phi)\right\} .
$$

A formula $\phi \in \operatorname{Disj}(\mathcal{A}, \mathcal{B})$ is a star formula if $\operatorname{br}(\phi)=\phi$.

It is easily seen that if $w=\left(A_{1}, B_{1}\right) \ldots\left(A_{k}, B_{k}\right)$, then $\operatorname{Branches}\left(\phi_{w}\right)=\left\{\left(A_{1}, \emptyset\right)\left(\emptyset, B_{1}\right) \ldots\left(A_{k}, \emptyset\right)\left(\emptyset, B_{k}\right)\right\}$, so $\operatorname{br}\left(\phi_{w}\right)=\phi_{w}$ and $\phi_{w}$ is a star formula. Similarly, if $\left\{w_{i} \mid i \in I\right\}$ is a finite subset of $(P(\mathcal{A}) \times P(\mathcal{B}))^{*}$, then $\bigvee_{i \in I} \phi_{w_{i}}$ is a star formula.

Lemma 40. For each $\phi \in \operatorname{Disj}(\mathcal{A}, \mathcal{B}), \operatorname{br}(\phi) \leq \phi$ and $\operatorname{Supp}(\operatorname{br}(\phi))=\operatorname{Supp}(\phi)$. 
Proof. A straightforward induction:

$$
\begin{aligned}
\operatorname{br}(x) & =\phi_{\epsilon}=x . \\
\operatorname{br}((\bigvee B) \vee \phi) & =\bigvee\left\{\phi_{(\emptyset, B)} \circ \phi_{w} \mid w \in \operatorname{Branches}(\phi)\right\} \\
& \leq \phi_{(\emptyset, B)} \circ\left(\bigvee\left\{\phi_{w} \mid w \in \operatorname{Branches}(\phi)\right\}\right) \leq \phi_{(\emptyset, B)} \circ \phi=(\bigvee B) \vee \phi . \\
\operatorname{br}([A] \phi) & =\bigvee\left\{\phi_{(A, \emptyset)} \circ \phi_{w} \mid w \in \operatorname{Branches}(\phi)\right\} \\
& \leq \phi_{(A, \emptyset)} \circ\left(\bigvee\left\{\phi_{w} \mid w \in \operatorname{Branches}(\phi)\right\}\right) \leq \phi_{(A, \emptyset)} \circ \phi=[A] \phi . \\
\operatorname{br}\left(\phi_{0} \vee \phi_{1}\right) & =\bigvee\left\{\phi_{w} \mid w \in \operatorname{Branches}\left(\phi_{0}\right)\right\} \cup\left\{\phi_{w} \mid w \in \operatorname{Branches}\left(\phi_{1}\right)\right\} \\
& =\operatorname{br}\left(\phi_{0}\right) \vee \operatorname{br}\left(\phi_{1}\right) \leq \phi_{0} \vee \phi_{1} .
\end{aligned}
$$

We come back now to our original goal, that of estimating upper bounds for formulas $\phi$ of the form $\phi=\bigwedge_{i \in I} \phi_{i}$ as in display (26), where now $\phi_{i} \in \operatorname{Disj}(\mathcal{A}, \mathcal{B})$ for each $i \in I$. The next Proposition reduces the problem of giving a closed expression for $\phi^{\rho(\phi)}$ and estimating an upper bound for the Ruitenburg number of $\phi$ as in (26) to that of a conjunction of star formulas, that is, formulas of the form

$$
\phi=\operatorname{def} \bigwedge_{i} \phi_{i}, \quad \text { with } \quad \phi_{i}={ }_{\operatorname{def}} \bigvee_{j \in J_{i}} \phi_{w_{i, j}} \text { and } w_{i, j} \in(P(\mathcal{A}) \times P(\mathcal{B}))^{*} .
$$

To understand how we shall use Proposition 41, recall that $\operatorname{Supp}\left(\phi_{i}\right)=\operatorname{Supp}\left(\operatorname{br}\left(\phi_{i}\right)\right)$ for all $i$; moreover, we shall show (Propositions 43 and 44 below) that $\bigwedge_{i} \phi_{\text {Supp }\left(\operatorname{br}\left(\phi_{i}\right)\right)} \leq\left(\bigwedge_{i} \operatorname{br}\left(\phi_{i}\right)\right)^{n}$ for $n$ large enough. These two facts entail

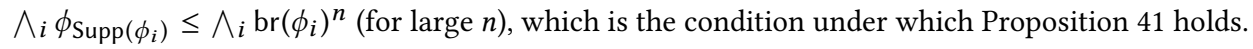

Proposition 41. Let I be a finite set, let $\phi_{i} \in \operatorname{Disj}(\mathcal{A}, \mathcal{B})$ for each $i \in I$, and let $n \geq 0$; suppose that $\bigwedge_{i} \phi_{\operatorname{Supp}}\left(\phi_{i}\right) \leq$ $\left(\bigwedge_{i} \operatorname{br}\left(\phi_{i}\right)\right)^{n}$. Then the following holds:

(i) $\bigwedge_{i} \phi_{\operatorname{Supp}\left(\phi_{i}\right)} \leq\left(\bigwedge_{i} \phi_{i}\right)^{n}$,

(ii) $\bigwedge_{i} \phi_{\mathrm{Supp}\left(\phi_{i}\right)}=\left(\bigwedge_{i} \phi_{i}\right)^{\rho\left(\bigwedge_{i} \phi_{i}\right)}$, and

(iii) $\rho\left(\bigwedge_{i} \phi_{i}\right) \leq \rho\left(\bigwedge_{i} \operatorname{br}\left(\phi_{i}\right)\right)$.

Proof. Statement (i) of the proposition follows from $\bigwedge_{i} \operatorname{br}\left(\phi_{i}\right) \leq \bigwedge_{i} \phi_{i}$, so $\left(\bigwedge_{i} \operatorname{br}\left(\phi_{i}\right)\right)^{n} \leq\left(\bigwedge_{i} \phi_{i}\right)^{n}$ and $\bigwedge_{i} \phi_{\operatorname{Supp}\left(\phi_{i}\right)} \leq$ $\left(\bigwedge_{i} \operatorname{br}\left(\phi_{i}\right)\right)^{n} \leq\left(\bigwedge_{i} \phi_{i}\right)^{n}$. We observe now that the relation $\bigwedge_{i} \phi_{\operatorname{Supp}\left(\phi_{i}\right)} \leq\left(\bigwedge_{i} \phi_{i}\right)^{n}$ implies

$$
\bigwedge_{i} \phi_{\operatorname{Supp}\left(\phi_{i}\right)}=\left(\bigwedge_{i} \phi_{i}\right)^{n}
$$

To this goal, it is enough to argue that $\left(\bigwedge_{i} \phi_{i}\right)^{n} \leq \bigwedge_{i} \phi_{\text {Supp }\left(\phi_{i}\right)}$, for each $n \geq 0$, which follows from $\left(\bigwedge_{i} \phi_{i}\right)^{n} \leq \phi_{i}^{n} \leq$ $\phi_{\text {Supp }\left(\phi_{i}\right)}^{n}=\phi_{\operatorname{Supp}\left(\phi_{i}\right)}$ (since $\phi_{\operatorname{Supp}\left(\phi_{i}\right)}$ is idempotent), for each $i \in I$.

Therefore, if $(i)$ holds, then $\bigwedge_{i} \phi_{\operatorname{Supp}\left(\phi_{i}\right)} \leq\left(\bigwedge_{i} \phi_{i}\right)^{n+1}$ as well, since $\left(\bigwedge_{i} \phi_{i}\right)^{n} \leq\left(\bigwedge_{i} \phi_{i}\right)^{n+1}$, and then

$$
\left(\bigwedge_{i} \phi_{i}\right)^{n+1}=\bigwedge_{i} \phi_{\operatorname{Supp}\left(\phi_{i}\right)}=\left(\bigwedge_{i} \phi_{i}\right)^{n}
$$

From these relations we immediately infer (ii). For (iii) we argue as follows. Let $K_{0}=\rho\left(\bigwedge_{i} \operatorname{br}\left(\phi_{i}\right)\right)$ and $K_{1}=\rho\left(\bigwedge_{i} \phi_{i}\right)$; since $\operatorname{Supp}\left(\phi_{i}\right)=\operatorname{Supp}\left(\operatorname{br}\left(\phi_{i}\right)\right)$, we also derive $\bigwedge_{i} \phi_{\operatorname{Supp}\left(\phi_{i}\right)}=\left(\bigwedge_{i} \operatorname{br}\left(\phi_{i}\right)\right)^{K_{0}}$ as an instance of (ii). The relation $K_{1} \leq K_{0}$ follows then by the inequalities

$$
\left(\bigwedge_{i} \operatorname{br}\left(\phi_{i}\right)\right)^{k} \leq\left(\bigwedge_{i} \phi_{i}\right)^{k} \leq \bigwedge_{i} \phi_{\operatorname{Supp}\left(\phi_{i}\right)}=\left(\bigwedge_{i} \phi_{i}\right)^{K_{1}}=\left(\bigwedge_{i} \operatorname{br}\left(\phi_{i}\right)\right)^{K_{0}}
$$

Manuscript submitted to ACM 
valid for any $k \geq 0$.

Let us give an explicit form to the iterates of a formula $\phi$ as in (34). To this goal, we shall assume that $J_{i}=\{1, \ldots, k\}=$ $[k]$ for each $i \in I$. We do not loose generality with this assumption, since the formula $\phi_{i}$ is equivalent to $\phi_{i} \vee \phi_{\epsilon}$. We shall make use of the distributive law (of disjunctions w.r.t. conjunctions) in the following form:

$$
\bigvee_{j \in[k]} \bigwedge_{i \in I} X_{j, i}=\bigwedge_{f:[k \longmapsto} \bigvee_{j \in[k]} X_{j, f(j)}
$$

Let us also introduce the following notation:

$$
\operatorname{Strat}_{n}=\operatorname{def} \prod_{1 \leq \ell \leq n} I^{[k]^{\ell-1}} .
$$

An element $f \in \operatorname{Strat}_{n}$ is a tuple $\left(f_{1}, \ldots, f_{n}\right)$ with $f_{\ell}:[k]^{\ell-1} \longrightarrow I$, for each $\ell=1, \ldots, n$. In particular, for $\ell=1$, we identify $f_{1} \in I^{[k]^{0}} \simeq I^{[1]}$ with an element of $I$. We think of a tuple $\left(f_{1}, \ldots, f_{n}\right) \in \operatorname{Strat}_{n}$ as a memory aware strategy for the first player of a two player game: the strategy tells him how to incrementally choose a tuple $\left(i_{1}, \ldots, i_{n}\right) \in I^{n}$ as a function of the opponent's choices $\left(j_{1}, \ldots j_{n-1}\right)$ (where $j_{\ell} \in[k]$ for $\left.\ell=1, \ldots, n-1\right)$, so $i_{\ell}=f_{\ell}\left(j_{1}, \ldots, j_{\ell-1}\right)$ for $\ell=1, \ldots, n$. We recall that there is a canonical bijection between $I \times \operatorname{Strat}_{n}^{[k]}$ and $\operatorname{Strat}_{n+1}$, as witnessed by the following computations:

$$
I \times \operatorname{Strat}_{n}^{[k]}=I \times\left(\prod_{1 \leq \ell \leq n} I^{[k]^{\ell-1}}\right)^{[k]} \simeq I^{[k]^{0}} \times \prod_{1 \leq \ell \leq n} I^{[k]^{\ell}} \simeq \text { Strat }_{n+1} .
$$

An explicit description of the bijection is as follows:

$$
\left(f_{0}, g\right) \in I \times \operatorname{Strat}_{n}^{[k]} \mapsto\left(f_{0}, h_{1}, \ldots, h_{n}\right) \in \operatorname{Strat}_{n+1},
$$

where for $\ell \geq 1$ we have

$$
h_{\ell}\left(j_{1}, j_{2}, \ldots, j_{\ell-1}\right)=\left[g\left(j_{1}\right)\right]_{\ell}\left(j_{2}, \ldots, j_{\ell-1}\right)
$$

Proposition 42. Let $\phi$ be of the form as in display (34). For each $n \geq 1$, we have

$$
\phi^{n}=\bigwedge_{\left(f_{1}, \ldots, f_{n}\right) \in \operatorname{Strat}_{n}} \bigvee_{j_{1}} \phi_{w_{f_{1}, j_{1}}}\left(\bigvee_{j_{2}} \phi_{w_{f_{2}\left(j_{1}\right), j_{2}}}\left(\ldots \bigvee_{j_{n}} \phi_{w_{f_{n}\left(j_{1}, j_{2} \ldots j_{n-1}\right), j_{n}}}\right)\right)
$$

In order to increase the readability, we shall write in the proof of the proposition and in the rest of this section $\phi_{i, j}$ in place of the more appropriate $\phi_{w_{i, j}}$.

Proof of Proposition 42. When $n=1$, then equation (36) reduces to

$$
\phi=\bigwedge_{f_{1} \in I} \bigvee_{j_{1} \in[k]} \phi_{f_{1}, j_{1}}
$$


so it holds simply by definition of $\phi$. Notice now that a word formula, and in particular each $\phi_{i, j}$, commutes with conjunctions; we use this fact in the inductive step. We suppose that (36) holds for $n \geq 1$ and compute as follows:

$$
\begin{aligned}
& \phi^{n+1}=\phi\left(\phi^{n}\right) \\
& =\bigwedge_{i_{0} \in I} \bigvee_{j_{0} \in[k]} \phi_{i_{0}, j_{0}}\left(\bigwedge_{f \in \operatorname{Strat}_{n}} \bigvee_{j_{1}} \phi_{f_{1}, j_{1}}\left(\bigvee_{j_{2}} \phi_{f_{2}\left(j_{1}\right), j_{2}}\left(\ldots \bigvee_{j_{n}} \phi_{f_{n}\left(j_{1}, j_{2} \ldots j_{n-1}\right), j_{n}}\right)\right)\right), \quad \text { by the inductive hypothesis, } \\
& =\bigwedge_{i_{0} \in I} \bigvee_{j_{0} \in[k]} \bigwedge_{f \in \text { Strat }_{n}} \phi_{i_{0}, j_{0}}\left(\bigvee_{j_{1}} \phi_{f_{1}, j_{1}}\left(\bigvee_{j_{2}} \phi_{f_{2}\left(j_{1}\right), j_{2}}\left(\ldots \bigvee_{j_{n}} \phi_{f_{n}\left(j_{1}, j_{2} \ldots j_{n-1}\right), j_{n}}\right)\right)\right) \text {, } \\
& \text { since } \phi_{i_{0}, j_{0}} \text { commutes with conjunctions, } \\
& =\bigwedge_{i_{0} \in I} \bigwedge_{g:\left[k \mapsto \text { Strat }_{n}\right.} \bigvee_{j_{0} \in[k]} \phi_{i_{0}, j_{0}}\left(\bigvee_{j_{1}} \phi_{g\left(j_{0}\right)_{1}, j_{1}}\left(\bigvee_{j_{2}} \phi_{g\left(j_{0}\right)_{2}\left(j_{1}\right), j_{2}}\left(\ldots \ldots \bigvee_{j_{n}} \phi_{g\left(j_{0}\right)_{n}\left(j_{1}, j_{2} \ldots j_{n-1}\right), j_{n}}\right)\right)\right), \quad \text { using }(35), \\
& =\bigwedge_{i_{0} \in I} \bigwedge_{g:\left[k \longmapsto \text { Strat }_{n}\right.} \bigvee_{j_{1}} \phi_{i_{0}, j_{1}}\left(\bigvee_{j_{2}} \phi_{g\left(j_{1}\right)_{1}, j_{2}}\left(\bigvee_{j_{3}} \phi_{g\left(j_{1}\right)_{2}\left(j_{2}\right), j_{3}}\left(\ldots \ldots \bigvee_{j_{n+1}} \phi_{g\left(j_{1}\right)_{n}\left(j_{2}, j_{3} \ldots j_{n}\right), j_{n+1}}\right)\right)\right) \\
& =\bigwedge_{h \in \text { Strat }_{n+1}} \bigvee_{j_{1}} \phi_{h_{1}, j_{1}}\left(\bigvee_{j_{2}} \phi_{h_{2}\left(j_{1}\right), j_{2}}\left(\bigvee_{j_{3}} \phi_{h_{3}\left(j_{1}, j_{2}\right), j_{3}}\left(\ldots \ldots \bigvee_{j_{n+1}} \phi_{h_{n+1}\left(j_{1}, j_{2}, j_{3} \ldots j_{n}\right), j_{n+1}}\right)\right)\right) \text {. }
\end{aligned}
$$

\subsection{A game for iterated conjunctions of star formulas}

Let $\phi=\bigwedge_{i \in I} \phi_{i}$ with $\phi_{i}=\bigvee_{j \in[k]} \phi_{w_{i, j}}$. For each $K \geq 1$, we describe next a two-player game $\mathcal{G}(\phi, K)$ (between Eve and Adam, and where Adam is the first player) with the following property: if Eve has a winning strategy in $\mathcal{G}(\phi, K)$, then the relation

$$
\bigwedge_{i \in I} \phi_{\operatorname{Supp}\left(\phi_{i}\right)} \leq \phi^{K}
$$

holds. Therefore, using Proposition 41, if Eve has a winning strategy in $\mathcal{G}(\phi, K)$, then $\phi^{\rho(\phi)}=\bigwedge_{i} \phi_{\operatorname{Supp}\left(\phi_{i}\right)}$ and that $\rho(\phi) \leq K$. Positions and moves of the game $\mathcal{G}(\phi, K)$ are as follows. Adam's positions are of the form $\left(i_{1}, j_{1}\right) \ldots\left(i_{n}, j_{n}\right)$, where $n \leq K$ and, for $\ell=1, \ldots, n, i_{\ell} \in I$ and $j_{\ell} \in[k]$. In such a position (when $n<K$ ) Adam chooses $i_{n+1} \in I$ and moves to the position $\left(i_{1}, j_{1}\right) \ldots\left(i_{n}, j_{n}\right)\left(i_{n+1}\right.$, ?). In this position Eve chooses $j_{n+1}$ and moves to $\left(i_{1}, j_{1}\right) \ldots\left(i_{n}, j_{n}\right)\left(i_{n+1}, j_{n+1}\right)$. The length of a position $\left(i_{1}, j_{1}\right) \ldots\left(i_{n}, j_{n}\right)$ is the integer $n$. The initial position is $\epsilon$ (the empty sequence or, in other words, the sequence of length $n=0)$.

To each of Adam's position $p=\left(i_{1}, j_{1}\right) \ldots\left(i_{k}, j_{k}\right)$, let $w_{p}=w_{i_{1}, j_{1}} \ldots w_{i_{k}, j_{k}}$. A terminal position $p=\left(i_{1}, j_{1}\right) \ldots\left(i_{K}, j_{K}\right)$ is a win for Eve (and a loss for Adam) if, for some $i \in I$, Supp $\left(\phi_{i}\right) \leq \phi_{w}$ with $w=w_{i_{1}, j_{1}} \ldots w_{i_{K}, j_{K}}$.

Proposition 43. If Eve has a winning strategy in the game $\mathcal{G}(\phi, K)$, then

$$
\bigwedge_{i \in I} \operatorname{Supp}\left(\phi_{i}\right) \leq \phi^{K}
$$

Proof. In view of (36) we need to show that, for any $f \in \operatorname{Strat}_{K}, \bigwedge_{i \in I} \operatorname{Supp}\left(\phi_{i}\right) \Vdash \oplus(f)$, where

$$
\oplus(f)=\operatorname{def} \bigvee_{j_{1}} \phi_{f_{1}, j_{1}}\left(\bigvee_{j_{2}} \phi_{f_{2}\left(j_{1}\right), j_{2}}\left(\ldots \bigvee_{j_{K}} \phi_{f_{K}\left(j_{1}, j_{2} \ldots j_{K-1}\right), j_{K}}\right)\right) .
$$

Let $f \in$ Strat $_{K}$ be fixed and observe that such an $f$ yields a strategy (not a winning one) for Adam in the game $\mathcal{G}(\phi, K)$. Now, if $g$ is a winning strategy for Eve in this game, then $f$ and $g$ determine a play $f \| g$ in the game such that, for some $i \in I, \phi_{\operatorname{Supp}\left(\phi_{i}\right)} \leq \phi_{w_{f \| g}}$. We have then $\bigwedge_{i} \phi_{\operatorname{Supp}\left(\phi_{i}\right)} \leq \phi_{\operatorname{Supp}\left(\phi_{i}\right)} \leq \phi_{w_{f \| g}} \leq \oplus(f)$.

$\operatorname{Recall}$ that $\operatorname{card}(\mathcal{A})=N$ and $\operatorname{card}(\mathcal{B})=M$. 
Proposition 44. Eve has a winning strategy in the game $\mathcal{G}(\phi,(N+1)(M+1))$.

Proof. Eve keeps a memory in order to decide how to move. Her memory is a pair $(A, B) \in P(\mathcal{A}) \times P(\mathcal{B})$ and, at the beginning of the play, $(A, B)=(\emptyset, \emptyset)$.

At each position $p=\left(i_{1}, j_{1}\right) \ldots\left(i_{\ell}, j_{\ell}\right)$ of the play, if the memory is $\left(A_{p}, B_{p}\right)$, then $A_{p}=\operatorname{Supp}_{\mathcal{A}}\left(w_{p}\right)$, where $w_{p}=w_{i_{1}, j_{1}} w_{i_{2}, j_{2}} \ldots w_{i_{\ell}, j_{\ell}}$. In particular, if $p^{\prime}$ is a prefix of $p$, then $A_{p^{\prime}} \subseteq A_{p}$. Moreover, if $w_{p}=w_{0} w_{1}$ with $w_{0}$ being the shortest prefix of $w_{p}$ such that $\operatorname{Supp}_{\mathcal{A}}\left(w_{0}\right)=A_{p}$, then $B_{p} \subseteq \operatorname{Supp}_{\mathcal{B}}\left(w_{1}\right)$. Notice that these conditions imply that $\left(A_{p}, B_{p}\right) \triangleleft w_{p}$, so $\phi_{\left(A_{p}, B_{p}\right)} \leq \phi_{w_{p}}$ by Lemma 39 .

Let $p=\left(i_{1}, j_{1}\right) \ldots\left(i_{\ell}, j_{\ell}\right)$. At position $p\left(i_{\ell+1}, ?\right)$, Eve chooses $j_{\ell+1}$ so that, if $p^{\prime}=p\left(i_{\ell+1}, j_{\ell+1}\right)$, Supp $\mathcal{A}_{\mathcal{A}}\left(w_{p^{\prime}}\right)$ is strictly greater than $A_{p}=\operatorname{Supp}_{\mathcal{A}}\left(w_{p}\right)$. If it is possible to choose such $j_{\ell+1}$, then she updates her memory to $\left(\operatorname{Supp}_{A}\left(w_{p^{\prime}}\right), \emptyset\right)$. Otherwise, if it is not possible to choose $j_{\ell+1}$ with these properties, then Eve chooses $j_{\ell+1}$ so $\operatorname{Supp}_{\mathcal{B}}\left(w_{i_{\ell+1}, j_{\ell+1}}\right)$ strictly includes $B_{p}$. She updates then the memory to $\left(A_{p^{\prime}}, B_{p^{\prime}}\right)=\left(A_{p}, B_{p} \cup \operatorname{Supp}_{\mathcal{B}}\left(\phi_{w_{i_{\ell+1}, j},{ }_{\ell+1}}\right)\right)$. If it is not possible to operate that kind of choices, then Eve chooses some $j_{\ell+1}$ at random and sets $\left(A_{p^{\prime}}, B_{p^{\prime}}\right)=\left(A_{p}, B_{p}\right)$.

Now, in a play, there are at most $N+1$ values for $A_{p}$ and, for each fixed $A_{p}$, there are at most $M+1$ values for $B_{p}$. Therefore, within $(N+1)(M+1)$ rounds either

(a) the play visits an Eve's position $p\left(i_{\ell+1}\right.$, ?) - therefore with $\ell<(N+1)(M+1)$ and $p$ of the form $\left(i_{1}, j_{1}\right) \ldots\left(i_{\ell}, j_{\ell}\right)-$ where she cannot extend $A_{p}$ nor $B_{p}$; that is, we have $\operatorname{Supp}_{\mathcal{A}}\left(w_{i_{\ell+1}, j}\right) \subseteq A_{p}$ and $\operatorname{Supp}_{\mathcal{B}}\left(w_{i_{\ell+1}, j}\right) \subseteq B_{p}$, for each $j \in[k]$; or

(b) the play ends up in an Adam's position $p=\left(i_{1}, j_{1}\right) \ldots\left(i_{\ell}, j_{\ell}\right)$ with $\ell=(N+1)(M+1)$, where now $A_{p}=\mathcal{A}$ and $B_{p}=\mathcal{B}$.

Suppose (a). Since $\operatorname{Supp}\left(\phi_{i_{\ell+1}}\right)=\left(\bigcup_{j} \operatorname{Supp}_{\mathcal{A}}\left(w_{i_{\ell+1}, j}\right), \cup_{j} \operatorname{Supp}_{\mathcal{A}}\left(w_{i_{\ell+1}, j}\right)\right)$, we have $\operatorname{Supp}_{\mathcal{A}}\left(\phi_{i_{\ell+1}}\right) \subseteq A_{p}$ and $\operatorname{Supp}_{\mathcal{B}}\left(\phi_{i_{\ell+1}}\right) \subseteq$ $B_{p}$. Since $\left(A_{p}, B_{p}\right) \triangleleft w_{p}$, it also follows that $\operatorname{Supp}\left(\phi_{i_{\ell+1}}\right) \triangleleft w_{p}$, so $\phi_{\operatorname{Supp}\left(\phi_{i_{\ell+1}}\right)} \leq \phi_{w_{p}}$ by Lemma 39. This shows that the position $p$ (as well as any of its extensions) is a win for Eve. If (b) then $A_{p}=\mathcal{A}$ and $B_{p}=\mathcal{B}$ so, in a similar way as before, we have $\phi_{\operatorname{Supp}\left(\phi_{i}\right)} \leq \phi_{w_{p}}$, this time for each $i \in I$.

We can now state the main result of this section.

Theorem 45. Let $\phi=\bigwedge_{i \in I} \phi_{i}$ where each $\phi_{i}$ is a disjunctive formula. Then $\rho(\phi) \leq(N+1)(M+1)$ where $N$ is the number of distinct head subformulas of $\phi$ and $M$ is the number of distinct side subformulas occurring in any of the $\phi_{i}$.

Proof. The statement holds iff and only if it holds when head and side subformulas of $\phi$ are propositional variables, that is, when $\phi_{i} \in \operatorname{Disj}(\mathcal{A}, \mathcal{B})$ for each $i \in I$, with $\operatorname{card}(\mathcal{A})=N$ and $\operatorname{card}(\mathcal{B})=M$. Moreover, according to Proposition 41, the statement of the theorem holds if $\bigwedge_{i \in I} \phi_{\operatorname{Supp}\left(\phi_{i}\right)} \leq \phi^{(N+1)(M+1)}$ and under the additional assumption that each $\phi_{i}$ is a star formula. Now the relation $\bigwedge_{i \in I} \phi_{\operatorname{Supp}\left(\phi_{i}\right)} \leq \phi^{(N+1)(M+1)}$ is a consequence of Proposition 44, stating that Eve has a winning strategy in the game $\mathcal{G}(\phi,(N+1)(M+1))$, and of Proposition 43, relating such a winning strategy to the relation.

Remark 46. The upper bound given in Theorem 45 appears to be orthogonal to bound implicit in Ruitenburg's paper [Ruitenburg 1984]. In the bound $\rho(\phi) \leq 2 n+2$, the size $n$ of $\phi$ is at least the number of implication subformulas of $\phi$. Now, in a formula of the form $\bigwedge_{i \in I} \phi_{i}$ with $\phi_{i} \in \operatorname{Disj}(\mathcal{A}, \mathcal{B})$, the number of implication subformulas might be exponentially larger than $N$ and $M$. Therefore the bound given in Theorem 45 is in this case tighter than Ruitenburg's bound. Conversely, we can derive from Theorem 45 a quadratic (in the size of the formula) upper bound for Ruitenburg's numbers of strongly positive formulas. This is achieved by considering that the size of a strongly positive formula is 
greater than the number of all the head and side subformulas in the conjuncts of its normal form (as in Lemma 14). Ruitenburg's upper bound is in this case tighter.

Remark 47. The following example shows that the quadratic upper bound is necessary, at least with respect to finding a winning strategy for Eve. Let $\mathcal{A}=$ def $\left\{\alpha_{1}, \ldots, \alpha_{N}\right\}$ and $\mathcal{B}=$ def $\left\{\beta_{1}, \ldots, \beta_{N}\right\}$. For each $k=1, \ldots, N$, let $P_{k}(\mathcal{B})$ be the set of subsets of $\mathcal{B}$ if size $k$. Let $I=\left\{(k, B) \mid B \in P_{k}(\mathcal{B})\right\}$ and, for each $(k, B) \in I$, consider the branch formula

$$
\phi_{(k, B)}=\operatorname{def} \bigvee_{\beta \in B} \phi_{\left(\left\{\alpha_{k}\right\},\{\beta\}\right)}
$$

Adam can use the following winning strategy in all the games $\mathcal{G}(\phi, K)$ with $K<\frac{N(N-1)}{2}$. He starts by choosing $(N, \mathcal{B})$ until Eve has chosen at least $N-1$ different symbols from $\mathcal{B}$. Let $\beta_{N}$ the only symbol not chosen by Eve. Then Adam chooses $\left(N-1, \mathcal{B} \backslash\left\{\beta_{N}\right\}\right)$ and iterates this choice until Eve has chosen exactly $N-2$ different symbols. Let $\beta_{N-1}$ be the only symbol from $\mathcal{B} \backslash\left\{\beta_{N}\right\}$ which has not been chosen by Eve, then Adam chooses $\left(N-2, \mathcal{B} \backslash\left\{\beta_{N}, \beta_{N-1}\right\}\right)$, and so on. Eve needs $N-1+(N-2)+(N-2)+\ldots$ rounds to win. This example raises the question of the completeness of the game: does the existence of an Adam's winning strategy in $\mathcal{G}(\phi, K)$ implies that $\bigwedge_{i \in I} \phi_{\operatorname{Supp}\left(\phi_{i}\right)} \nless \leq \phi^{K}$ ?

Remark 48. We considered

$$
\phi_{n}(x)={ }_{\text {def }} \bigvee_{i=1, \ldots, n} \alpha_{i} \rightarrow\left(\beta_{i} \vee x\right)
$$

and used fCube [Ferrari et al. 2010] to compute the values of $\operatorname{cl}\left(\phi_{n}\right)$ and $\rho\left(\phi_{n}\right)$. For $n \in\{2,3,4,5\}$, we obtained that $\operatorname{cl}\left(\phi_{n}\right)=\rho\left(\phi_{n}\right)=n+1$. This raises the question whether there is any strongly positive formula of the IPC for which we have $\operatorname{cl}\left(\phi_{n}\right)<\rho\left(\phi_{n}\right)$.

\section{A CONSTANT UPPER BOUND FOR DISJUNCTIONS OF ALMOST-TOPOLOGIES}

In this Section we exemplify how investigating (lower bounds of) closure ordinals might lead to uncover non-trivial properties of Heyting algebras. Example 21 illustrated the elimination procedure in the case of weakly negative formula-terms. It considered a formula-term of the form

$$
\phi(x)={ }_{\operatorname{def}} \bigvee_{i \in I}\left(x \rightarrow b_{i}\right) \rightarrow a_{i},
$$

where the index set was a two element set. In view of the similarity of these formulas with the disjunctive formulas of Section 6, we conjectured that closure ordinals of formulas as the ones above increase as the size of $I$ becomes larger-so to exhibit tightness of the upper bound on closure ordinals of weakly negative formula-terms presented in Proposition 29. Yet, all our automatized tests, for which we used the tool fCube [Ferrari et al. 2010], pointed towards the opposite direction. We finally managed to disprove the conjecture: all these formula-terms converge to their least fixed-points in 3 steps.

Let $H$ be a Heyting algebra. For $a, b \in H$, we call $j_{a, b}$ defined by

$$
j_{a, b}(x)=_{\operatorname{def}}(x \rightarrow a) \rightarrow b,
$$

an almost-topology (briefly, an atop). The reason is the following: when $a=b$, then $j_{a, a}$ is a closure operator (that is, a monotone inflating idempotent function on $H$ ); more than that, it is a Lawvere-Tierney topology or nucleus, see [Johnstone 1982, Chapter II, section 2], meaning that they are strong: $x \wedge j_{a, a}(y) \leq j_{a, a}(x \wedge y)$, for each $x, y \in H$. We Manuscript submitted to ACM 
shall consider disjunctions of atops, for which we need a convenient notation: for a family of pairs $\Pi=\left\{\left(a_{i}, b_{i}\right) \mid i \in I\right\}$, we shall write

$$
\phi_{\Pi}(x)=\operatorname{def} \bigvee_{i \in I} j_{a_{i}, b_{i}}(x)
$$

\subsection{Elementary properties of atops}

In the following, we use $[a, b]$ to denote the closed interval $\{x \mid a \leq x \leq b\}$.

Lemma 49. The following holds, for each $x \in H$ :

(i) $x \leq j_{a, b}(x)$ if and only if $x \leq a \rightarrow b$. In particular, if $a \leq b$, then $x \leq j_{a, b}(x)$;

(ii) $j_{a, b}^{2}(x)=j_{a, b}(x)$ if and only $j_{a, b}(b) \leq j_{a, b}(x)$. In particular, this holds when $b \leq x$.

Consequently, the restriction of $j_{a, b}$ to the interval $[b, a \rightarrow b]$ is a closure operator.

Proof. (i) $x \leq(x \rightarrow a) \rightarrow b$ iff $x \wedge x \rightarrow a \leq b$, iff $x \wedge a \leq b$, iff $x \leq a \rightarrow b$. For the second statement, notice that if $a \leq b$, then $x \leq \mathrm{\top}=a \rightarrow b$.

(ii) Notice firstly that the condition $j_{a, b}^{2}(x)=j_{a, b}(x)$ is equivalent to $j_{a, b}^{2}(x) \leq j_{a, b}(x)$. As a matter of fact, $j_{a, b}(x) \leq a \rightarrow b$ for each $x \in H$ so by (i) we always have $j_{a, b}(x) \leq j_{a, b}^{2}(x)$.

We prove that $j_{a, b}^{2}(x) \leq j_{a, b}(x)$ is equivalent to $j_{a, b}(b) \leq j_{a, b}(x)$. By repeated use of compatibility, we have the following equality:

$$
\begin{aligned}
j_{a, b}^{2}(x) \wedge(x \rightarrow a)=j_{a, b}((x \rightarrow a) \rightarrow b) \wedge(x \rightarrow a) & =j_{a, b}(((x \rightarrow a) \rightarrow b) \wedge(x \rightarrow a)) \wedge(x \rightarrow a), \\
& =j_{a, b}(b \wedge(x \rightarrow a)) \wedge(x \rightarrow a)=j_{a, b}(b) \wedge(x \rightarrow a) .
\end{aligned}
$$

It follows that

$$
j_{a, b}^{2}(x) \leq j_{a, b}(x) \quad \text { iff } \quad j_{a, b}(b) \wedge(x \rightarrow a)=j_{a, b}^{2}(x) \wedge(x \rightarrow a) \leq b \quad \text { iff } \quad j_{a, b}(b) \leq j_{a, b}(x) .
$$

Finally, if $b \leq x$, then $j_{a, b}(b) \leq j_{a, b}(x)$ so $j_{a, b}^{2}(x)=j_{a, b}(x)$.

Since $j_{a, b}(\perp)=b, j_{a, b}(\top)=a \rightarrow b$, and $j_{a, b}$ is monotone, we also remark:

Lemma 50. The image of $H$ via $j_{a, b}$ is contained in the interval $[b, a \rightarrow b]$.

Thus we have $j_{a, b}(x)=j_{a, b}(x) \wedge(a \rightarrow b)$. We shall exploit this fact many times, in conjunction with strongness. The following Lemma exemplifies this.

Lemma 51. If $f: H \longrightarrow H$ is a strong monotone mapping, then $j_{a, b}(f(x)) \leq j_{a, b}\left(f\left(j_{a, b}(x)\right)\right)$.

Proof. We compute as follows:

$$
\begin{array}{rlr}
j_{a, b}(f(x)) & =j_{a, b}(f(x)) \wedge(a \rightarrow b), & \text { by Lemma 50, } \\
& =j_{a, b}(f(x \wedge(a \rightarrow b))) \wedge(a \rightarrow b), & \text { since } j_{a, b} \circ f \text { is strong, } \\
& \leq j_{a, b}\left(f\left(j_{a, b}(x \wedge(a \rightarrow b))\right) \wedge(a \rightarrow b),\right. & \text { using Lemma 49.(i) and the fact that } x \wedge(a \rightarrow b) \leq(a \rightarrow b), \\
& =j_{a, b}\left(f\left(j_{a, b}(x)\right)\right),
\end{array}
$$

where in the last step we have again used Lemma 50 and the fact that $j_{a, b} \circ f$ is strong. 
To end this Section, it is useful to pinpoint two identities that shall be useful later. The first one is obtained by repeatedly using compatibility of $j_{a, b}$ :

$$
j_{a, b}(x) \wedge c=j_{a \wedge c, b \wedge c}(x \wedge c) \wedge c=j_{a \wedge c, b \wedge c}(x) \wedge c .
$$

In particular, since $j_{a, b}(x)=j_{a, b}(x) \wedge(a \rightarrow b)$, we derive

$$
j_{a, b}(x)=j_{a \wedge b, b}(x) \wedge(a \rightarrow b) .
$$

The latter identity relates a general atop to a specific atop $j_{a, b}$ with the property that $a \leq b$ which-according to Lemma 49.(i)-is always inflating.

\subsection{Closure of prefixed-points of strong monotone mappings under exponentiation}

The following Lemma asserts that prefixed-points of strong monotone mappings are closed under exponentiation. This property seems to be the hidden principal ingredient in the proof of the main result of this section, Theorem 55.

Lemma 52. Let $g: H \longrightarrow H$ be a strong monotone mapping. If $c \in$ Pre $_{g}$, then $x \rightarrow c \in$ Pre $_{g}$, for each $x, c \in H$.

Proof. The Lemma is an immediate consequence of equation (5): $g(x \rightarrow c) \leq x \rightarrow g(c) \leq x \rightarrow c$, when $c \in \operatorname{Pre}_{g}$.

We shall study next when $j_{a, b}(x)=j_{a, c}(x)$. Indeed, in view of Lemma 52 , we shall have that $j_{a, b}(x)$ is a prefixed point of a strong $g$, if this equality holds and $c$ is a prefixed-point of $g$.

Lemma 53. We have $j_{a, b}(x)=j_{a, c}(x)$ if and only if $x \rightarrow a \leq b \leftrightarrow c$. In particular, if $b \leq c \leq x \leq a \rightarrow b$, then $j_{a, b}(x)=j_{a, c}(x)$.

Proof. By symmetry, it will be enough to prove that $j_{a, b}(x) \leq j_{a, c}(x)$ if and only if $x \rightarrow a \leq b \rightarrow c$.

Suppose that $x \rightarrow a \leq b \rightarrow c$. Then

$$
((x \rightarrow a) \rightarrow b) \wedge(x \rightarrow a)=b \wedge(x \rightarrow a) \leq b \wedge(b \rightarrow c) \leq c
$$

so $j_{a, b}(x) \leq j_{a, c}(x)$. Conversely, suppose that $j_{a, b}(x) \leq j_{a, c}(x)$. Then

$$
b \wedge(x \rightarrow a)=((x \rightarrow a) \rightarrow b) \wedge(x \rightarrow a) \leq c,
$$

so $x \rightarrow a \leq b \rightarrow c$.

For the last sentence, we can use the characterization we have just given. Suppose $b \leq c \leq x \leq a \rightarrow b$. Then $x \rightarrow a \leq \top=b \rightarrow c$. Also $c \wedge x \rightarrow a \leq x \wedge x \rightarrow a=x \wedge a \leq a \rightarrow b \wedge a \leq b$, so $x \rightarrow a \leq c \rightarrow b$.

Proposition 54. Let $g$ be a strong monotone mapping. If $c \in \operatorname{Pre}_{g} \cap[f, e \rightarrow f]$, then $j_{e, f}(x) \in \operatorname{Pre}_{g}$ for each $x \in[c, e \rightarrow f]$.

Proof. By the previous Corollary, we can write $j_{e, f}(x)=j_{e, c}(x)$. It follows then from Lemma 52 that $j_{e, f}(x)=$ $j_{e, c}(x) \in \operatorname{Pre}_{g}$.

\subsection{Convergence in 3 steps for disjunctions of atops}

Let therefore $\Pi=\left\{\left(a_{i}, b_{i}\right) \mid i \in I\right\}$ be fixed; in order to improve readability, let us put, for each $i \in I$,

$$
j_{i}(x)=_{\text {def }} j_{a_{i}, b_{i}}(x) .
$$

Manuscript submitted to ACM 
THEOREM 55. The function $\phi_{\Pi}$ defined as in equation (37) converges to its least fixed-point in 3 steps.

Proof. We need to prove that $j_{k}\left(\phi_{\Pi}^{3}(\perp)\right) \leq \phi_{\Pi}^{3}(\perp)$, for each $k \in I$. If we put $b={ }_{\text {def }} \phi_{\Pi}(\perp)=\bigvee_{i \in I} b_{i}$ then we need to show that

$$
j_{k}\left(\phi_{\Pi}^{2}(b)\right) \leq \phi_{\Pi}^{2}(b) \quad \text { for each } k \in I .
$$

Let, from now on, $k \in I$ be fixed and put

$$
J_{k}(x)={ }_{\text {def }} j_{a_{k} \wedge b_{k}, b_{k}}(x),
$$

so $j_{k}(x)=J_{k}(x) \wedge\left(a_{k} \rightarrow b_{k}\right)$ as from equation 38. We shall argue that, for each $i \in I$, the following relation holds:

$$
j_{i}\left(J_{k}\left(\phi_{\Pi}(b)\right)\right) \leq J_{k}\left(\phi_{\Pi}(b)\right) .
$$

Once equation (40) is proved, we prove (39) as follows:

$$
\begin{array}{rlr}
j_{k}\left(\phi_{\Pi}^{2}(b)\right) & =j_{k}\left(\bigvee_{i \in I} j_{i}\left(\phi_{\Pi}(b)\right)\right) \leq j_{k}\left(\bigvee_{i \in I} j_{i}\left(J_{k}\left(\phi_{\Pi}(b)\right)\right)\right), & \text { by Lemma 51, } \\
& \leq j_{k}\left(\bigvee_{i \in I} J_{k}\left(\phi_{\Pi}(b)\right)\right)=j_{k}\left(J_{k}\left(\phi_{\Pi}(b)\right)\right), & \text { using equation (40), } \\
& =j_{k}\left(j_{k}\left(\phi_{\Pi}(b)\right)\right), & \text { since of } j_{k}(x)=j_{k}(x) \wedge\left(a_{k} \rightarrow b_{k}\right) \text { and } j_{k} \text { is strong, } \\
& =j_{k}\left(\phi_{\Pi}(b)\right), & \text { using } b_{k} \leq b \leq \phi_{\Pi}(b) \text { and Lemma 49.(ii), } \\
& \leq \phi_{\Pi}^{2}(b) .
\end{array}
$$

In order to prove that equation (40) holds, we use Proposition 54 and argue that a certain $j_{e, f}(x)$ is a prefixed-point of $j_{i}$. Let, in the statement of the Proposition,

$$
e=\text { def } a_{k} \wedge b_{k} \wedge\left(a_{i} \rightarrow b_{i}\right), \quad f=\text { def } b_{k} \wedge\left(a_{i} \rightarrow b_{i}\right), \quad c=\text { def } j_{i}(b), \quad x==_{\text {def }} \phi_{\Pi}(b) \wedge\left(a_{i} \rightarrow b_{i}\right), \quad g=j_{i} .
$$

To apply the Proposition, we need to verify that $(i) f \leq c \leq x \leq e \rightarrow f$ and that $(i i) c$ is a prefixed-point of $j_{i}$.

(i) We have $b \wedge a_{i} \rightarrow b_{i} \leq a_{i} \rightarrow b_{i}$ and therefore, by Lemma 49.(i),

$$
b \wedge\left(a_{i} \rightarrow b_{i}\right) \leq j_{i}\left(b \wedge\left(a_{i} \rightarrow b_{i}\right)\right)=j_{i}(b) \wedge\left(a_{i} \rightarrow b_{i}\right)=j_{i}(b) .
$$

Using this relation, we see that

$$
\begin{aligned}
f=b_{k} \wedge\left(a_{i} \rightarrow b_{i}\right) & \leq b \wedge\left(a_{i} \rightarrow b_{i}\right) \\
& \leq j_{i}(b)=c \\
& \leq \phi_{\Pi}(b) \wedge\left(a_{i} \rightarrow b_{i}\right)=x \\
& \leq \top=e \rightarrow f .
\end{aligned}
$$

(ii) From $b_{i} \leq b$ and Lemma 49.(ii) it immediately follows that $c=j_{i}(b)$ is a prefixed-point of $j_{i}$.

From $(i),(i i)$ and Proposition 54, it follows that $j_{e, f}(x)$ is a prefixed-point of $j_{i}$. Recall now that

$$
\begin{aligned}
x & =\phi_{\Pi}(b) \wedge\left(a_{i} \rightarrow b_{i}\right), \\
j_{e, f}(y) & =j_{a_{k} \wedge b_{k} \wedge\left(a_{i} \rightarrow b_{i}\right), b_{k} \wedge\left(a_{i} \rightarrow b_{i}\right)}(c) \wedge\left(a_{i} \rightarrow b_{i}\right)=J_{k}(y) \wedge\left(a_{i} \rightarrow b_{i}\right), \quad \text { for each } y \in H .
\end{aligned}
$$


We have therefore

$$
\begin{aligned}
j_{i}\left(J_{k}\left(\phi_{\Pi}(b)\right)\right. & =j_{i}\left(J_{k}\left(\phi_{\Pi}(b) \wedge\left(a_{i} \rightarrow b_{i}\right)\right) \wedge\left(a_{i} \rightarrow b_{i}\right)\right) \wedge\left(a_{i} \rightarrow b_{i}\right) \\
& =j_{i}\left(j_{e, f}\left(\phi_{\Pi}(b) \wedge\left(a_{i} \rightarrow b_{i}\right)\right)\right) \\
& \leq j_{e, f}\left(\phi_{\Pi}(b) \wedge\left(a_{i} \rightarrow b_{i}\right)\right) \leq J_{k}\left(\phi_{\Pi}(b)\right),
\end{aligned}
$$

proving relation (40).

Remark 56. The above upper bound is tight. Recall that $I$ is the index set over the disjunction by which $\phi_{\Pi}$ is defined, see (37), so $\operatorname{card}(I)$ is the number of atops being joined. Computations with fCube [Ferrari et al. 2010] show that $\mathrm{cl}\left(\phi_{\Pi}\right)=2$ when $\operatorname{card}(I)=1$, and that $\operatorname{cl}\left(\phi_{\Pi}\right)=3$ when $\operatorname{card}(I) \in\{2,3,4,5\}$. Quite interestingly we obtained the same pattern for Ruitenburg's numbers: $\rho\left(\phi_{\Pi}\right)=\operatorname{cl}\left(\phi_{\Pi}\right)$ when $\operatorname{card}(I) \in\{2,3,4,5\}$. This raises the question whether the results presented in this section can be lifted to Ruitenburg's number; more generally and also considering Remark 48 , the question whether there is any formula $\phi \in \mathcal{F}_{\text {IPC }}$ for which $\operatorname{cl}(\phi)<\rho(\phi)$ is open.

\section{CONCLUSIONS}

By Ruitenburg's theorem [Ruitenburg 1984] the least and the greatest fixed-point of a monotone polynomial on an Heyting algebra are constructible by means of finite Kleene's iterations. The number of iterations needed to reach the fixed-point is computable from the formula defining the polynomial, and therefore the least and greatest fixed-points are definable within the Intuitionistic Propositional Calculus. We have emphasized in Sections 4 and 5 that definability of these fixed-points is a consequence of the important structural properties of the IPC, the deduction theorem and the existence of bisimulation quantifiers [Ghilardi and Zawadowski 2011; Pitts 1992]. The main contribution of the first part of this paper is a new perspective on these fixed-points, alternative to the usual Kleene's iteration, that yields both an axiomatization of these fixed-points and a fixed-point elimination procedure (described in Section 6). Moreover,

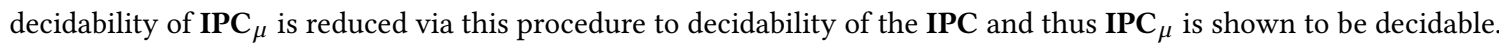
The second part of this paper analyses back the fixed-point elimination procedure from the point of view of Kleene's iteration: in Section 7 upper bounds of the finite closure ordinals of formulas of the IPC are systematically discovered; since they are not in general tight, in Section 8 the critical formulas that arise from the elimination procedure have been further investigated leading to better approximations (which also apply to Ruitenburg's number of these formulas). The complexity of a general investigation of the finite closure ordinals of intuitionistic formulas is witnessed in Section 9 where we exhibit a large class of formulas whose least fixed-point are surprisingly reached in 3 steps.

The second part of this paper is, in our opinion, a rich source of open problems susceptible to grow up to a research domain that we might name the arithmetic of the IPC. Ruitenburg's theorem is in this context a cornerstone and, for this reason, an alternative proof of this result was obtained in [Ghilardi and Santocanale 2018, 2019]. In This proof, using the duality for finitely presented Heyting algebras developed in [Ghilardi and Zawadowski 2011], yields new perspectives, yet the approximations of Ruitenburg's numbers so obtained are again far from being optimal. A deeper understanding of Ruitenburg's theorem might come from the results presented in Section 8 where-as in Ruitenburg's original proof-the notion of inflating function plays a key role.

Beside Intuitionistic Logic, we conjecture that many of the general results presented in Section 7 generalize to infinite closure ordinals. It is therefore conceivable that they might be of use in current research on closure ordinals for modal $\mu$-calculi, see e.g. [Afshari and Leigh 2013; Czarnecki 2010; Gouveia and Santocanale 2017]. Finally, one more question worth to be answered is to what extent the results presented in this paper can be transferred back to the modal $\mu$-calculi Manuscript submitted to ACM 
on restricted classes of models whose existing literature [Alberucci and Facchini 2009a,b; D’Agostino and Lenzi 2010] motivated and contributed to advance the present research.

\section{REFERENCES}

Bahareh Afshari and Graham E. Leigh. 2013. On closure ordinals for the modal $\mu$-calculus. In Computer science logic 2013. LIPIcs. Leibniz Int. Proc. Inform., Vol. 23. Schloss Dagstuhl. Leibniz-Zent. Inform., Wadern, 30-44.

Luca Alberucci and Alessandro Facchini. 2009a. The Modal $\mu$-Calculus Hierarchy on Restricted Classes of Transition Systems. The fournal of Symbolic Logic 74, 4 (2009), 1367-1400.

Luca Alberucci and Alessandro Facchini. 2009b. On Modal $\mu$-Calculus and Gödel-Löb Logic. Studia Logica 91, 2 (2009), $145-169$.

André Arnold and Damian Niwiński. 2001. Rudiments of $\mu$-calculus. Studies in Logic and the Foundations of Mathematics, Vol. 146. Elsevier.

Nathalie Bertrand and Philippe Schnoebelen. 2013. Computable fixpoints in well-structured symbolic model checking. Formal Methods in System Design 43, 2 (2013), 233-267.

Nick Bezhanishvili and Dick de Jongh. 2006. Intuitionistic Logic. Technical Report. Institute for Logic, Language and Computation, Universiteit van Amsterdam. PP-2006-25. Available at http://www.illc.uva.nl/Research/Publications/Reports/PP-2006-25.text.pdf.

Willem J. Blok, Peter Köhler, and Don Pigozzi. 1984. On the structure of varieties with equationally definable principal congruences II. Algebra Universalis 18, 3 (1984), 334-379.

Stephen L. Bloom and Zoltán Ésik. 1993. Iteration Theories: The Equational Logic of Iterative Processes. Springer-Verlag New York, Inc., New York, NY, USA. Pierre Clairambault. 2013. Strong functors and interleaving fixpoints in game semantics. RAIRO - Theor. Inf. and Applic. 47, 1 (2013), 25-68.

Robin Cockett and Dwight Spencer. 1995. Strong Categorical Datatypes II: A Term Logic for Categorical Programming. Theor. Comput. Sci. 139, 1\&2 (1995), 69-113.

Marek Czarnecki. 2010. How Fast Can the Fixpoints in Modal $\mu$-Calculus Be Reached?. In 7th Workshop on Fixed Points in Computer Science, FICS 2010, L. Santocanale (Ed.). Brno, Czech Republic, 89. Available from Hal: https://hal.archives-ouvertes.fr/hal-00512377.

Giovanna D’Agostino and Marco Hollenberg. 2000. Logical Questions Concerning the mu-Calculus: Interpolation, Lyndon and Los-Tarski. f. Symb. Log. $65,1(2000), 310-332$.

Giovanna D’Agostino and Giacomo Lenzi. 2010. On the $\mu$-calculus over transitive and finite transitive frames. Theor. Comput. Sci. 411, 50 (2010), $4273-4290$.

Mads Dam and Dilian Gurov. 2002. $\mu$-Calculus with Explicit Points and Approximations. Fournal of Logic and Computation 12, 2 (2002), $255-269$.

Frithjof Dau. 2006. Some Notes on Proofs with Alpha Graphs. In Conceptual Structures: Inspiration and Application, 14th International Conference on Conceptual Structures, ICCS 2006, Aalborg, Denmark, fuly 16-21, 2006, Proceedings (Lecture Notes in Computer Science), Henrik Schärfe, Pascal Hitzler, and Peter Øhrstrøm (Eds.), Vol. 4068. Springer, 172-188.

Mauro Ferrari, Camillo Fiorentini, and Guido Fiorino. 2010. fCube: An Efficient Prover for Intuitionistic Propositional Logic. In Logic for Programming, Artificial Intelligence, and Reasoning, Christian G. Fermüller and Andrei Voronkov (Eds.). Springer Berlin Heidelberg, Berlin, Heidelberg, $294-301$.

Sabine Frittella and Luigi Santocanale. 2014. Fixed-Point Theory in the Varieties $\mathcal{D}_{n}$. In Relational and Algebraic Methods in Computer Science - 14th International Conference, RAMiCS 2014, Marienstatt, Germany, April 28-May 1, 2014. Proceedings (Lecture Notes in Computer Science), Peter Höfner, Peter Jipsen, Wolfram Kahl, and Martin Eric Müller (Eds.), Vol. 8428. Springer, 446-462.

Gerhard Gentzen. 1935. Untersuchungen über das logische Schließen. I. Mathematische Zeitschrift 39, 1 (1935), 176-210.

Silvio Ghilardi, Maria João Gouveia, and Luigi Santocanale. 2016. Fixed-point elimination in the intuitionistic propositional calculus. In Foundations of software science and computation structures. Lecture Notes in Comput. Sci., Vol. 9634. Springer, Berlin, 126-141.

Silvio Ghilardi and Luigi Santocanale. 2018. Ruitenburg's theorem via duality and bounded bisimulations. In Advances in modal logic. Vol. 12. Coll. Publ., $277-290$.

Silvio Ghilardi and Luigi Santocanale. 2019. Free Heyting Algebra Endomorphisms: Ruitenburg's Theorem and Beyond. (Jan. 2019). preprint. Available fron Hal: https://hal.archives-ouvertes.fr/hal-01969235.

Silvio Ghilardi and Marek Zawadowski. 1997. Model Completions, r-Heyting Categories. Ann. Pure Appl. Logic 88, 1 (1997), $27-46$.

Silvio Ghilardi and Marek Zawadowski. 2011. Sheaves, Games, and Model Completions: A Categorical Approach to Nonclassical Propositional Logics (1st ed.). Springer Publishing Company, Incorporated.

Maria João Gouveia and Luigi Santocanale. 2017. $\aleph_{1}$ and the modal $\mu$-calculus. In Computer science logic 2017. LIPIcs. Leibniz Int. Proc. Inform., Vol. 82. Schloss Dagstuhl. Leibniz-Zent. Inform., Wadern, Art. No. 38, 16.

David Harel, Dexter Kozen, and Jerzy Tiuryn. 2000. Dynamic logic. MIT Press, Cambridge, MA. xvi+459 pages.

Peter Johnstone. 1982. Stone Spaces. Number 3 in Cambridge Studies in Advanced Mathematics. Cambridge University Press.

G. Max Kelly. 1982. Basic Concepts of Enriched Category Theory. Number 64 in Lecture Notes in Mathematics. Cambridge University Press. Republished in: Reprints in Theory and Applications of Categories, No. 10 (2005) pp. 1-13.

Anders Kock. 1972. Strong Functors and Monoidal Monads. Archiv der Mathematik XXIII (1972), 113-120.

Dexter Kozen. 1983. Results on the Propositional mu-Calculus. Theor. Comput. Sci. 27 (1983), 333-354. 
Marcus Kracht. 2006. Modal Consequence Relations. In Handbook of Modal Logic, Volume 3 (Studies in Logic and Practical Reasoning), Patrick Blackburn, Johan F. A. K. van Benthem, and Frank Wolter (Eds.). Elsevier Science Inc., New York, NY, USA, Chapter 8, 491-547.

Karoliina Lehtinen and Sandra Quickert. 2015. Deciding the First Levels of the Modal mu Alternation Hierarchy by Formula Construction. In 24th EACSL Annual Conference on Computer Science Logic, CSL 2015, September 7-10, 2015, Berlin, Germany (LIPIcs), Stephan Kreutzer (Ed.), Vol. 41. Schloss Dagstuhl - Leibniz-Zentrum fuer Informatik, 457-471.

Sergey Mardaev. 1993. Least fixed points in Grzegorczyk's Logic and in the Intuitionistic Propositional Logic. Algebra and Logic 32, 5 (1993), $279-288$. Sergey Mardaev. 1994. Convergence of positive schemes in S4 and Int. Algebra and Logic 33, 2 (1994), 95-101.

Sergey Mardaev. 2007. Definable fixed points in modal and temporal logics - a survey. Fournal of Applied Non-Classical Logics 17, 3 (2007), $317-346$. Sara Negri and Jan von Plato. 2001. Structural proof theory. Cambridge University Press, Cambridge. xviii +257 pages.

Andrew M. Pitts. 1992. On an Interpretation of Second Order Quantification in First Order Intuitionistic Propositional Logic. 7. Symb. Log. 57, 1 (1992), 33-52.

Wim Ruitenburg. 1984. On the Period of Sequences $\left(A^{n}(p)\right)$ in Intuitionistic Propositional Calculus. The fournal of Symbolic Logic 49, 3 (1984), 892-899. Luigi Santocanale. 2002. $\mu$-bicomplete categories and parity games. Theor. Inform. Appl. 36, 2 (2002), 195-227. 